\title{
Throughput Maximization for Cognitive Radio Networks Using Active Cooperation and Superposition Coding
}

\author{
Doha Hamza, Member, IEEE, Ki-Hong Park, Member, IEEE, Mohamed-Slim Alouini, Fellow, IEEE and Sonia \\ Aïssa, Senior Member, IEEE
}

\begin{abstract}
We propose a three-message superposition coding scheme in a cognitive radio relay network exploiting active cooperation between primary and secondary users. The primary user is motivated to cooperate by substantial benefits it can reap from this access scenario. Specifically, the time resource is split into three transmission phases: The first two phases are dedicated to primary communication, while the third phase is for the secondary's transmission. We formulate two throughput maximization problems for the secondary network subject to primary user rate constraints and per-node power constraints with respect to the time durations of primary transmission and the transmit power of the primary and the secondary users. The first throughput maximization problem assumes a partial power constraint such that the secondary power dedicated to primary cooperation, i.e. for the first two communication phases, is fixed apriori. In the second throughput maximization problem, a total power constraint is assumed over the three phases of communication. The two problems are difficult to solve analytically when the relaying channel gains are strictly greater than each other and strictly greater than the direct link channel gain. However, mathematically tractable lowerbound and upperbound solutions can be attained for the two problems. For both problems, by only using the lowerbound solution, we demonstrate significant throughput gains for both the primary and the secondary users through this active cooperation scheme. We find that most of the throughput gains come from minimizing the second phase transmission time since the secondary nodes assist the primary communication during this phase. Finally, we demonstrate the superiority of our proposed scheme compared to a number of reference schemes that include best relay selection, dual-hop routing, and an interference channel model.
\end{abstract}

\section{INTRODUCTION}

Cognitive radio technology has the potential to resolve spectrum scarcity and to maximize spectral efficiency [1]. Many techniques exist on methods for the coexistence of the primary and the secondary users of the network as long as the secondary users do not degrade the performance of the primary network [2]-[4]. One of the popular techniques for

Manuscript received May, 14, 2014; revised October, 24, 2014; accepted January, 28, 2015. The review of this paper was coordinated by $\mathrm{Z}$. Wang.

D. Hamza, K-H. Park and M-S. Alouini are with the Computer, Electrical, and Mathematical Science and Engineering (CEMSE) Division, King Abdullah University of Science and Technology (KAUST), Thuwal, Makkah Province, Kingdom of Saudi Arabia. Emails: \{doha.hamzamohamed, kihong.park, slim.alouini\}@kaust.edu.sa.

S. Aïssa is with the Institut National de la Recherche Scientifique (INRSEMT), University of Quebec, Montreal, QC, Canada. Email: aissa@emt.inrs.ca

The work of M. -S. Alouini was supported by the Qatar National Research Fund (a member of Qatar Foundation) under NPRP Grant NPRP 5-250-2-087. The statements made herein are solely the responsibility of the authors. the secondary users to gain access to the wireless medium is through relaying the primary signal so that certain primary quality of service requirements such as rate and outage probability constraints are met [5]-[8].

Within the realm of cooperative communication, superposition coding has been known to improve the region of the achievable rates for collaborative wireless users [9], [10]. For example, the works in [11], [12] show two-level superposition coding to be superior to simple decode-and-forward of the original message. Naturally, because of its benefits, the use of superposition coding was also adopted in cognitive relaying [13], [14].

Whether superposition coding is employed or not, the use of cognitive relaying is predicated on the primary user playing an active role in spectrum management. Such role was shown to benefit both users [15], [16]. Hence, we also focus in this work on a communication scheme whereby the primary users are not oblivious to the presence of the secondary users but play an active and cooperative role to maximize their benefit from this cognitive spectrum access.

There have also been previous works on cooperative primary/secondary communications. In [7], when the secondary user cooperates, time is divided into alternating time slots. In odd slots, the primary user transmits its data, while in even slots the secondary user transmits a superimposed signal of its message and that of the primary user. The design objective is to find the optimal secondary power to maximize the secondary throughput without degrading primary performance. The primary and secondary time slots are assumed to be fixed in that work. In [8], active cooperation is proposed within a game-theoretic framework so that the users optimize the time durations of primary and secondary communications for fixed powers at both transmitters. In [11], time is split into two transmission phases. In the first time phase, the primary user broadcasts its message, while in the second time phase, the secondary user broadcasts its signal superimposed on the primary signal. The authors optimize the time and power allocations so that the secondary throughput is maximized while the primary rate is fixed at the direct-link rate. The system resembles the two-hop scheme of [17] which we will investigate in the numerical results section. In [12], on the otherhand, a multiple access (MAC) scheme is considered with two primary users sending to a common destination. Network coding and superposition coding are utilized at the cognitive node that superimposes its own message on network-coded primary signals. However, the MAC channel model in [12] is different from the model we consider here.

In this paper, we analyze a half-duplex coding scheme 
for cooperative communication between the primary node and two cognitive nodes to send the primary message to a destination. Through superposition coding, the primary user splits its message into three parts and transmits in two phases, while the secondary users utilize a third transmission phase for their own communication. By actively relaying the primary message in the second transmission phase, the secondary node aims to maximize its benefit from this spectrum access model. We formulate two throughput maximization problems for the secondary network subject to the primary user rate requirements and the power constraints of all nodes in the network. The optimization variables are the time durations of the communication phases and the transmit powers for these phases at the primary and secondary users.

In the first problem, which we first introduced in [18], the secondary nodes pre-allocate a designated portion of their powers to relay the primary message in the second communication phase. By actively optimizing the primary power allocation and the time duration of each transmission phase, we maximize the time left for secondary transmission. In the second throughput problem, we use a total secondary power constraint and consider not only the time resource left for secondary operation but also the power resource.

Owing to the highly non-linear nature of the equations involving the optimization variables, we demonstrate the difficulty associated with finding analytical expressions for the time and power portions. However, by proposing lowerbound and upperbound solutions, we find mathematically tractable expressions that are readily implementable. Even by considering the lowerbound solution, we can demonstrate the superiority of the proposed schemes to existing works in the literature.

The main contributions of our work are as follows: i) We provide simple lowerbound and upperbound analytical solutions for the formulated optimization problems; ii) Our work provides a more generalized framework than [7], [8] for the active cooperation between primary and secondary users, since we optimize both time durations and powers for the transmission phases; and iii) Compared to other reference schemes, our scheme is shown to provide better throughput for the secondary users without degrading primary performance. To the contrary, for example, we show that our suggested scheme provides more throughput for the primary node than is the achievable rate via a direct link alone (no cooperation) while achieving anon-zero throughput for the secondary nodes.

It is noted that we assume perfect knowledge of the channel coefficients among the different nodes. This is not uncommon in the information-theoretic literature. For example, both models in [13] and [19] work under the assumption that the instantaneous channel gains are perfectly known. Furthermore, these two references suggest practical ways to acquire the channel knowledge. For example, [13] suggests the use of the CTS/RTS messages that are typically exchanged between the primary source and destination to estimate the channels $h_{p s}$ and $h_{s d}$. All other channel gains can be included in a conditionfor-allowing-secondary-transmission (CST) message which is broadcast by the primary network. Most of those channel estimation techniques may require some form of coordination between the primary and secondary nodes. However, given the substantial benefits both users can reap from our proposed access model, it is reasonable to expect such form of coordination between the different nodes in order to disseminate the channel state information (CSI) efficiently and promptly.

We also note that there are two ways by which the nodes can calculate the optimization variables in the two optimization problems we formulate. Either each node has all the available CSI to make its own calculations in a decentralized way or each node sends CSI to a central controller, for example the primary destination, which in turn calculates the optimized values and then broadcasts such values to all the other nodes. We lean towards the second solution as the different nodes may have varied computational powers and it is best to relegate such computational task to the most capable node. Under such case, the nodes may share the CSI through some of the mini-slots on a dedicated primary control channels as suggested in [20]. The interested reader is requested to refer to the aforementioned reference for more details on the mechanism for sharing such information and the required overhead.

The remainder of this paper is organized as follows. Section II presents the system model and communication scheme. We formulate and solve the first optimization problem with a partial power constraint in Section III. Then in Section IV, the optimization problem with a total power constraint is solved. Section V presents some illustrative numerical results along with comparisons to reference schemes. Finally, we conclude the paper in Section VI.

\section{SYSTEM MODEL}

We consider the spectrum sharing network illustrated in Fig. 1. The network comprises a primary user (P) seeking to communicate with its destination (D) in the presence of a cognitive pair, the secondary source $(S)$ and receiver $(R)$, who communicate with each other. We consider DF relaying such that both cognitive nodes , $\mathrm{S}$ and $\mathrm{R}$, assist in relaying the primary message in exchange for spectrum access for their own communication.

More specifically, we model a half-duplex channel with time division such that transmission is carried out in three phases as shown in Fig. 1. In the first transmission phase, of duration $\alpha_{1}$, the primary source broadcasts its message. Both $\mathbf{S}$ and $\mathrm{R}$ attempt to decode their intended messages of the primary signal while the destination node D keeps its received message to decode later. In the second transmission phase, of duration $\alpha_{2}$, the primary broadcasts another message and the cognitive nodes forward the messages they decoded in the first phase to the destination node D. At the end of this second phase, the destination decodes the [rimary messages received over two phases. The third transmission phase, of duration $1-\alpha_{1}-\alpha_{2}$, is reserved for the communication between the cognitive nodes $\mathrm{S}$ and $\mathrm{R}$.

In this work, we concern ourselves with maximizing the throughput of the secondary nodes, i.e. maximizing the time duration and power of the third transmission phase remaining for cognitive operation, provided that the primary node is guaranteed to obtain at least its direct-link rate. 


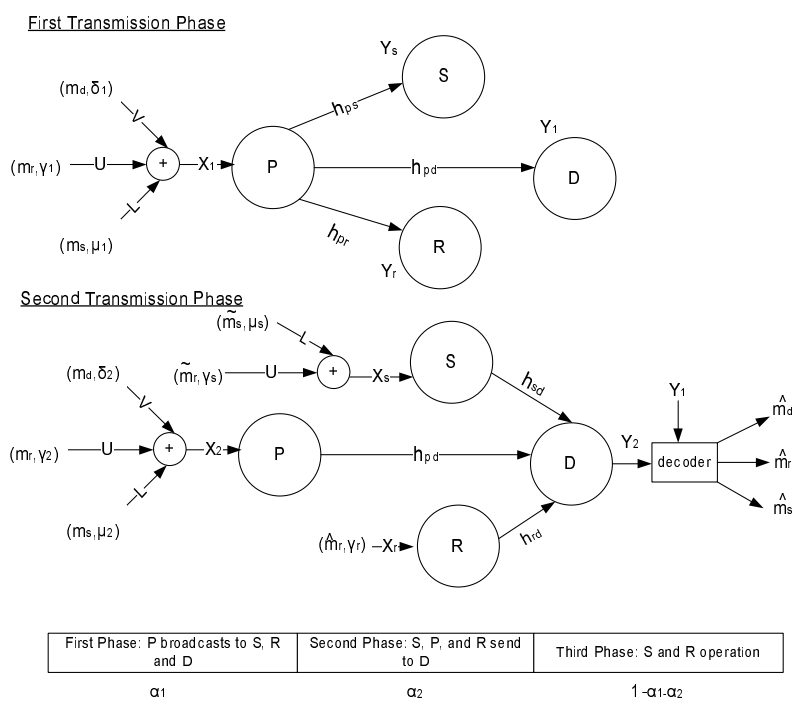

Fig. 1. Proposed scheme. In the first phase, node P broadcasts a superimposed message to all nodes in the network. Nodes $\mathrm{S}$ and $\mathrm{R}$ decode their intended messages after this first phase. During the second phase, node $\mathrm{P}$ broadcasts another superimposed message, while nodes $\mathrm{S}$ and $\mathrm{R}$ forward their decoded messages from the previous phase. Node D decodes the primary messages received over two phases. The third phase is reserved for the cognitive nodes.

\section{A. Three-Message Superposition Coding Communication Scheme}

The considered channel is memoryless and has finite input alphabets $\mathcal{X}, \mathcal{X}_{s}$ and $\mathcal{X}_{r}$ corresponding to the primary source, secondary source and secondary receiver, respectively. The three channel output alphabets are $\mathcal{Y}, \mathcal{Y}_{s}$ and $\mathcal{Y}_{r}$ corresponding to the primary destination, secondary source and secondary receiver, respectively. The channel is specified by a conditional distribution $p\left(y, y_{r}, y_{s} \mid x, x_{r}, x_{s}\right)$. A code $\left(2^{n R_{p}}, n\right)$ for this channel consists of a message set $\mathcal{M}=1,2, \ldots, 2^{n R_{p}}$, three encoders at nodes $\mathrm{P}, \mathrm{S}$ and $\mathrm{R}$ and three decoders at $\mathrm{D}, \mathrm{S}$ and $\mathrm{R}$.

We consider complex Gaussian channels where $h_{i j}$ denotes the channel gain between node $i \in\{p, s, r\}$ and node $j \in$ $\{d, s, r\}$. In this subsection, we focus on the communication scheme when $\left|h_{p s}\right|>\left|h_{p r}\right|>\left|h_{p d}\right|$ to illustrate the basic idea. The extension to other channel conditions will be considered in Section III.

Under such channel circumstances, to send a message $m$ of rate $R_{p}$ to the destination, the primary divides $m$ into three parts, $\left(m_{d}, m_{r}, m_{s}\right)$ with rates $R_{d}, R_{r}$ and $R_{s}$, respectively, where $R_{d}+R_{r}+R_{s}=R_{p}$. Message $m_{d}$ is decoded at the destination after the second phase, while message $m_{r}$ is intended to be relayed by $\mathrm{R}$. Since $\left|h_{p s}\right|>\left|h_{p r}\right|>\left|h_{p d}\right|$, message $\left(m_{r}, m_{s}\right)$ is intended to be relayed by $\mathrm{S}$ so that the rate at $\mathrm{S}$ is $R_{S}=R_{s}+R_{r}$. Let us denote the codeword as $x^{\alpha_{1} n}=$ $\left[x_{1}, x_{2}, \ldots, x_{\alpha_{1} n}\right]$ and $x^{\alpha_{2} n}=\left[x_{\alpha_{1} n+1}, x_{\alpha_{1} n+2}, \ldots, x_{\left(\alpha_{1}+\alpha 2\right) n}\right]$.

1) Codebook Generation: Fix $p(u) p\left(x_{r} \mid u\right) p(l) p\left(x_{s} \mid u, l\right)$ $p\left(x \mid u, l, x_{r}, x_{s}\right)$. Generate $2^{n R_{r}}$ independent and identically distributed (i.i.d.) sequences $u^{n}\left(m_{r}\right) \sim \prod_{i=1}^{n} p\left(u_{i}\right)$. Then, for each $u^{n}$, generate one sequence $x_{r}^{n}\left(m_{r}\right) \sim \prod_{i=1}^{n} p\left(x_{r i} \mid u_{i}\right)$. Generate $2^{n R_{s}}$ i.i.d. sequences $l^{n}\left(m_{s}\right) \sim \prod_{i=1}^{n} p\left(l_{i}\right)$.
For each $l^{n}$ and each $u^{n}$, generate one sequence $x_{s}^{n}\left(m_{s}, m_{r}\right) \sim \prod_{i=1}^{n} p\left(x_{s i} \mid u_{i}, l_{i}\right)$. Finally, for each $\left(u^{n}\left(m_{r}\right), x_{r}^{n}\left(m_{r}\right), l^{n}\left(m_{s}\right), x_{s}^{n}\left(m_{s}, m_{r}\right)\right) \quad$ sequence, we generate $2^{n R_{d}}$ sequences $x^{n}\left(m_{d}, m_{r}, m_{s}\right) \sim$ $\prod_{i=1}^{n} p\left(x_{i} \mid u_{i}, x_{r i}, l_{i}, x_{s i}\right)$.

To send a message $m$, node $\mathrm{P}$ maps it to the codeword $x^{n}\left(m_{d}, m_{r}, m_{s}\right) \in \mathcal{X}$. During the first phase, $\mathrm{P}$ sends $x^{\alpha_{1} n}$, while $\mathbf{R}$ and $\mathrm{S}$ listen. $\mathrm{R}$ and $\mathrm{S}$ then decode $\tilde{m}_{r}$ and $\left(\hat{m}_{s}, \hat{m}_{r}\right)$, respectively, from the received signal and re-encode it into the codewords $x_{r}^{n}\left(\tilde{m}_{r}\right)$ and $x_{s}^{n}\left(\hat{m}_{s}, \hat{m}_{r}\right)$, respectively. During the second phase, $\mathrm{P}$ sends $x^{\alpha_{2} n}$, R sends $x_{r}^{\alpha_{2} n}$ and $\mathrm{S}$ sends $x_{s}^{\alpha_{2} n}$.

2) Decoding Technique: Under joint typicality decoding, at the end of phase 1 , node $\mathrm{R}$ chooses the unique $m_{r}$ such that,

$$
\left(Y_{r}^{\alpha_{1} n}, U^{\alpha_{1} n}\left(m_{r}\right)\right) \in \mathcal{A}_{\epsilon}^{\alpha_{1} n} .
$$

Otherwise, an error is declared. In the above equation, we refer to $\mathcal{A}_{\epsilon}^{\alpha_{1} n}$ as the set of jointly typical sequences $\left\{Y_{r}^{\alpha_{1} n}, U^{\alpha_{1} n}\left(m_{r}\right)\right\}$ with respect to the distribution $p\left(u\left(m_{r}\right), y_{r}\right)$, i.e. the set of $\alpha_{1} n$-sequences with empirical entropies $\epsilon$-close to the true entropies [21]. ${ }^{1}$ During the same phase, phase 1 , node $\mathrm{S}$ also chooses the unique $\left(m_{r}, m_{s}\right)$ such that,

$$
\left(Y_{s}^{\alpha_{1} n}, L^{\alpha_{1} n}\left(m_{s}\right), U^{\alpha_{1} n}\left(m_{r}\right)\right) \in \mathcal{A}_{\epsilon}^{\alpha_{1} n} .
$$

Otherwise, an error is declared.

On the other hand, the destination applies joint decoding using the signal received during both transmission phases, and chooses the unique $\left(m_{d}, m_{r}, m_{s}\right)$ such that

$$
\left(Y_{1}^{\alpha_{1} n}, U^{\alpha_{1} n}\left(m_{r}\right), L^{\alpha_{1} n}\left(m_{s}\right), X^{\alpha_{1} n}\left(m_{d}, m_{r}, m_{s}\right)\right) \in \mathcal{A}_{\epsilon}^{\alpha_{1} n}
$$

and

$$
\begin{array}{r}
\left(Y_{2}^{\alpha_{2} n}, U^{\alpha_{2} n}\left(m_{r}\right), L^{\alpha_{2} n}\left(m_{s}\right), X^{\alpha_{2} n}\left(m_{d}, m_{r}, m_{s}\right)\right. \\
\left., X_{r}^{\alpha_{2} n}\left(m_{r}\right), X_{s}^{\alpha_{2} n}\left(m_{s}, m_{r}\right)\right) \in \mathcal{A}_{\epsilon}^{\alpha_{2} n} .
\end{array}
$$

Otherwise, an error is declared. Accordingly, the rate satisfying the following constraints is achievable.

Proposition 1 : All rates satisfying

$$
\begin{aligned}
R_{p} & \leq \alpha_{1} I\left(Y_{r} ; U\right)+\alpha_{1} I\left(Y_{s} ; L \mid U\right)+\alpha_{1} I\left(Y_{1} ; X \mid U, L\right) \\
& +\alpha_{2} I\left(Y_{2} ; X \mid U, L, X_{r}, X_{s}\right) \\
R_{p} & \leq \alpha_{1} I\left(Y_{s} ; U, L\right)+\alpha_{1} I\left(Y_{1} ; X \mid U, L\right) \\
& +\alpha_{2} I\left(Y_{2} ; X \mid U, L, X_{r}, X_{s}\right) \\
R_{p} & \leq \alpha_{1} I\left(Y_{1} ; U, L, X\right)+\alpha_{2} I\left(Y_{2} ; U, L, X, X_{r}, X_{s}\right) \\
R_{p} & \leq \alpha_{1} I\left(Y_{s} ; U L\right)+\alpha_{1} I\left(Y_{1} ; L, X \mid U\right) \\
& +\alpha_{2} I\left(Y_{2} ; L, X, X_{s} \mid U, X_{r}\right) \\
R_{p} & \leq \alpha_{1} I\left(Y_{r} ; U\right)+\alpha_{1} I\left(Y_{1} ; L, X \mid U\right) \\
& +\alpha_{2} I\left(Y_{2} ; L, X, X_{s} \mid U, X_{r}\right)
\end{aligned}
$$

are achievable for some joint distribution $p(u) p\left(x_{r} \mid u\right) p(l)$ $p\left(x_{s} \mid u, l\right) p\left(x \mid u, l, x_{r}, x_{s}\right) p\left(y, y_{r}, y_{s} \mid x, x_{r}, x_{s}\right)$.

Proof: We apply joint typicality arguments to characterize the error events at the decoders. The analysis is detailed in Appendix A.

\footnotetext{
${ }^{1}$ With a slight abuse of notation, we use the same symbol $\mathcal{A}_{\epsilon}$ to denote the different sets of jointly typical sequences where the relevant joint distributions are implicitly understood.
} 


\section{B. Communication Scheme for Gaussian Signaling and AWGN} Channels

In this subsection, we specialize the rate equations to the case of Gaussian signaling and AWGN channels. The received signals over the two transmission phases, as depicted in Fig. 1, can be written as:

$$
\begin{aligned}
& Y_{1}=h_{p d} X_{1}+Z_{1} \\
& Y_{r}=h_{p r} X_{1}+Z_{r} \\
& Y_{s}=h_{p s} X_{1}+Z_{s} \\
& Y_{2}=h_{p d} X_{2}+h_{r d} X_{r}+h_{s d} X_{s}+Z_{2}
\end{aligned}
$$

where $X=\left[X_{1}^{\alpha_{1} n} X_{2}^{\alpha_{2} n}\right], X_{r}$ and $X_{s}$ are the transmitted signals by $\mathrm{P}, \mathrm{R}$ and $\mathrm{S}$, respectively, and where $Z_{1}, Z_{2}, Z_{r}$ and $Z_{s}$ are independent AWGNs with variance $N$. Nodes P, R and $\mathrm{S}$ have individual power budgets, $P_{p}, P_{r}$ and $P_{s}$, respectively. The transmitted signals can be written as:

$$
\begin{aligned}
& X_{1}=\sqrt{\delta_{1} P_{p}} V+\sqrt{\gamma_{1} P_{p}} U+\sqrt{\mu_{1} P_{p}} L, \\
& X_{2}=\sqrt{\delta_{2} P_{p}} V+\sqrt{\gamma_{2} P_{p}} U+\sqrt{\mu_{2} P_{p}} L, \\
& X_{r}=\sqrt{\gamma_{r} P_{r}} U, \\
& X_{s}=\sqrt{\mu_{s} P_{s}} L+\sqrt{\gamma_{s} P_{s}} U,
\end{aligned}
$$

where $V\left(m_{d}\right), U\left(m_{r}\right)$ and $L\left(m_{s}\right) \sim \mathcal{N}(0,1)$ are independent. The terms $\delta_{1}$ and $\delta_{2}$ are the power portions of node $\mathrm{P}$ allocated to $m_{d}$ in the first and second phase, respectively, while $\gamma_{1}$ and $\gamma_{2}$ are the power portions allocated by $\mathrm{P}$ to $m_{r}$ in the first and second phases, and $\mu_{1}$ and $\mu_{2}$ are the power portions of $\mathrm{P}$ allocated to $m_{s}$ in the first and second phases. Also, node $\mathrm{R}$ uses $\gamma_{r}$ of its power to forward $\tilde{m}_{r}$ to the destination, while $\mathbf{S}$ uses $\mu_{s}$ power to forward $\hat{m}_{s}$ and $\gamma_{s}$ power to forward $\hat{m}_{r}$. The primary's power portions must satisfy constraints such that,

$$
\alpha_{1}\left(\delta_{1}+\gamma_{1}+\mu_{1}\right) P_{p}+\alpha_{2}\left(\delta_{2}+\gamma_{2}+\mu_{2}\right) P_{p} \leq P_{p} .
$$

The secondary source and receiver also have power constraints. We will apply two distinct power constraints for the secondary network in the next two sections. Based on the above modeling, we have the following corollary.

Corollary 1 : For AWGN channels, all rates satisfying (19), (20), and (21) are achievable.

Proof: The rates are derived by evaluating (5), (6), (7), (8) and (9) for the input in (14), (15), (16) and (17). When specializing to the Gaussian signaling case, (6) is greater than (7), while (9) is greater than (8), hence we are left with three inequalities.

\section{COMmunication SCHEME For Throughrut Maximization With a PaRtial Power Budget}

In this section, we formulate the secondary throughput maximization problem as follows:

$$
\begin{array}{cl}
\min _{\omega} & \alpha_{1}+\alpha_{2}, \\
\text { s.t. } & I_{1} \geq R_{p}, \quad I_{2} \geq R_{p}, \quad I_{3} \geq R_{p}, \\
& R_{p} \geq \log _{2}\left(1+\frac{\left|h_{p d}\right|^{2} P_{p}}{N}\right)=R_{d l}, \\
& \alpha_{1}\left(\delta_{1}+\gamma_{1}+\mu_{1}\right)+\alpha_{2}\left(\delta_{2}+\gamma_{2}+\mu_{2}\right) \leq 1, \\
& \alpha_{2} \gamma_{r} P_{r}^{p} \leq P_{r}^{p}, \alpha_{2}\left(\mu_{s}+\gamma_{s}\right) P_{s}^{p} \leq P_{s}^{p},
\end{array}
$$

where $\omega=\left[\begin{array}{lllllllllll}\alpha_{1} & \alpha_{2} & \delta_{1} & \delta_{2} & \gamma_{1} & \gamma_{2} & \mu_{1} & \mu_{2} & \gamma_{r} & \gamma_{s} & \mu_{s}\end{array}\right]$ is the vector of optimization variables. The objective of minimizing $\alpha_{1}+\alpha_{2}$ is equivalent to maximizing the secondary throughput. The first three constraints come from the rate equations of (19)(21). The fourth constraint guarantees a minimum rate for the primary user, which is at least equal to the direct-link rate, $R_{d l} .^{2}$ The remaining constraints are power constraints, where in addition to the primary power constraint of (18), we added partial power constraints for $\mathrm{S}$ and $\mathrm{R}$. Note that we consider a partial power constraint wherein $\mathrm{S}$ and $\mathrm{R}$ each pre-allocate power portions of their total powers, respectively $P_{s}^{p}$ and $P_{r}^{p}$, to relay the primary message in the second communication phase. This means, $P_{s}^{p} \leq P_{s}$ and $P_{r}^{p} \leq P_{r}$, where $P_{s}$ and $P_{r}$ are the total power budgets for $\mathrm{S}$ and $\mathrm{R}$, respectively. ${ }^{3}$ For the case of $\left|h_{p s}\right|>\left|h_{p r}\right|>\left|h_{p d}\right|$, it can be shown that the rate constraints are highly non-linear in the optimization variables. This makes a closed form solution mathematically intractable. However, as shown in Appendix B, we can state the following lowerbound proposition for the solution of the above optimization problem.

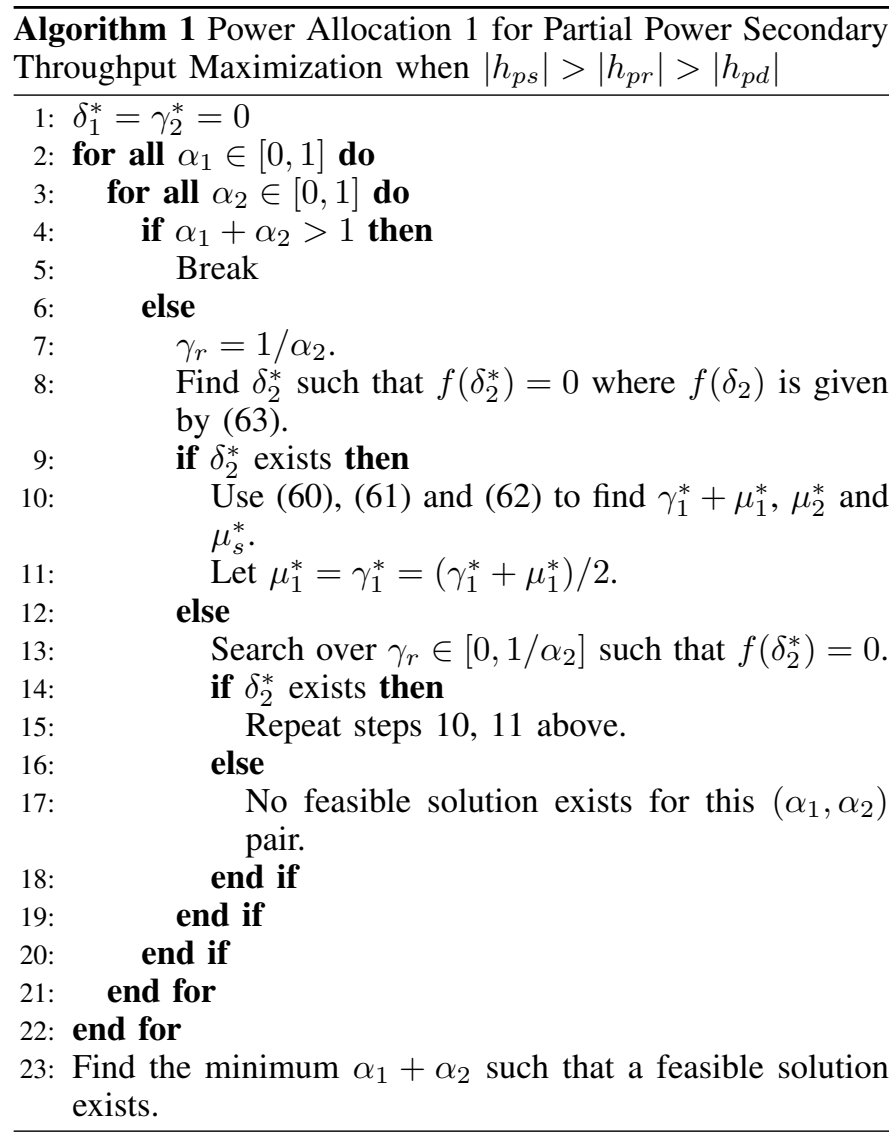

Proposition 2 : The primary's transmitter splits its message

\footnotetext{
${ }^{2}$ This constraint can be adjusted to achieve rates higher than the direct link alone as we show in the numerical results section.

${ }^{3}$ In the next section, we consider the optimization problem with the total power budgets.
} 
$R_{p} \leq \alpha_{1} \log _{2}\left(1+\frac{\left|h_{p r}\right|^{2} \gamma_{1} P_{p}}{N+\left|h_{p r}\right|^{2} P_{p}\left(\delta_{1}+\mu_{1}\right)}\right)+\alpha_{1} \log _{2}\left(1+\frac{\left|h_{p s}\right|^{2} \mu_{1} P_{p}}{N+\left|h_{p s}\right|^{2} P_{p} \delta_{1}}\right)+\alpha_{1} \log _{2}\left(1+\frac{\left|h_{p d}\right|^{2} \delta_{1} P_{p}}{N}\right)+\alpha_{2} \log _{2}\left(1+\frac{\left|h_{p d}\right|^{2} \delta_{2} P_{p}}{N}\right)=I_{1}$

$R_{p} \leq \alpha_{1} \log _{2}\left(1+\frac{\left|h_{p d}\right|^{2} P_{p}\left(\delta_{1}+\gamma_{1}+\mu_{1}\right)}{N}\right)$

$+\alpha_{2} \log _{2}\left(1+\frac{\left|h_{p d}\right|^{2} P_{p}\left(\delta_{2}+\gamma_{2}+\mu_{2}\right)+\left|h_{r d}\right|^{2} P_{r} \gamma_{r}+\left|h_{s d}\right|^{2} P_{s}\left(\mu_{s}+\gamma_{s}\right)+\zeta}{N}\right)=I_{2}$,

$R_{p} \leq \alpha_{1} \log _{2}\left(1+\frac{\left|h_{p r}\right|^{2} P_{p} \gamma_{1}}{N+\left|h_{p r}\right|^{2} P_{p}\left(\delta_{1}+\mu_{1}\right)}\right)+\alpha_{1} \log _{2}\left(1+\frac{\left|h_{p d}\right|^{2} P_{p}\left(\delta_{1}+\mu_{1}\right)}{N}\right)$

$+\alpha_{2} \log _{2}\left(1+\frac{\left|h_{p d}\right|^{2} P_{p}\left(\delta_{2}+\mu_{2}\right)+\left|h_{s d}\right|^{2} P_{s} \mu_{s}+2 \sqrt{\left|h_{p d}\right|^{2}\left|h_{s d}\right|^{2} \mu_{s} \mu_{2} P_{p} P_{s}}}{N}\right)=I_{3}$,

where $\zeta=2 \sqrt{\left|h_{r d}\right|^{2}\left|h_{p d}\right|^{2} \gamma_{r} \gamma_{2} P_{r} P_{p}}+2 \sqrt{\left|h_{r d}\right|^{2}\left|h_{s d}\right|^{2} \gamma_{r} \gamma_{s} P_{r} P_{s}}+2 \sqrt{\left|h_{s d}\right|^{2}\left|h_{p d}\right|^{2} \gamma_{s} \gamma_{2} P_{s} P_{p}}+2 \sqrt{\left|h_{s d}\right|^{2}\left|h_{p d}\right|^{2} \mu_{s} \mu_{2} P_{s} P_{p}}$.

according to the channel conditions as follows:

1) If $\left|h_{p s}\right|>\left|h_{p r}\right|>\left|h_{p d}\right|$, P splits its message into three parts, $m_{d}, m_{r}$ and $m_{s}$, as summarized in Algorithm 1 or $P$ splits its message into two parts, $m_{d}$ and $m_{r}$, as summarized in Algorithm 2. The attainable throughput is the maximum of the output of Algorithms 1 and 2.

2) If $\left|h_{p s}\right|>\left|h_{p d}\right|>\left|h_{p r}\right|$, $P$ splits its message into $m_{d}$ and $m_{s}$ only and uses Algorithm 1 with $\gamma_{1}=\gamma_{s}=\gamma_{r}=0$.

3) If $\left|h_{p d}\right|>\left|h_{p s}\right|>\left|h_{p r}\right|$ or $\left|h_{p d}\right|>\left|h_{p r}\right|>\left|h_{p s}\right|$, P sends only $m_{d}$ through the direct communication link and the cognitive throughput is zero.

4) The case of $\left|h_{p r}\right|>\left|h_{p s}\right|>\left|h_{p d}\right|$ corresponds to case 1 above, with the roles of $S$ and $R$ reversed so that $R$ sends $\left(m_{s}, m_{r}\right)$ while $S$ sends $m_{s}$ only.

5) The case of $\left|h_{p r}\right|>\left|h_{p d}\right|>\left|h_{p s}\right|$ corresponds to case 2 above, with the roles of $S$ and $R$ reversed so that $R$ sends $m_{r}$ and $S$ is silent.

Proof: See Appendix B.

In the numerical results section, we evaluate the performance of the proposed algorithm in comparison with other existing schemes in the literature. Next, we consider a similar optimization problem with total power constraints.

\section{COMmunication Scheme For Throughrut MAXimization WiTh A TOTAL POWER Budget}

In this problem, we consider the total powers that the cognitive nodes will have for all three communication phases, hence the throughput maximization problem is formulated as

$$
\begin{aligned}
\max _{\omega} & \left(1-\alpha_{1}-\alpha_{2}\right) \log _{2}\left(1+\frac{\left|h_{s}\right|^{2} P_{s} \delta_{s}}{N}\right), \\
\text { s.t. } & I_{1} \geq R_{p}, \quad I_{2} \geq R_{p}, \quad I_{3} \geq R_{p}, \\
& R_{p} \geq \log _{2}\left(1+\frac{\left|h_{p d}\right|^{2} P_{p}}{N}\right), \\
& \alpha_{1}\left(\delta_{1}+\gamma_{1}+\mu_{1}\right)+\alpha_{2}\left(\delta_{2}+\gamma_{2}+\mu_{2}\right) \leq 1, \\
& \alpha_{2}\left(\gamma_{s}+\mu_{s}\right) P_{s}+\left(1-\alpha_{1}-\alpha_{2}\right) \delta_{s} P_{s} \leq P_{s}, \\
& \alpha_{2} \gamma_{r} P_{r} \leq P_{r},
\end{aligned}
$$

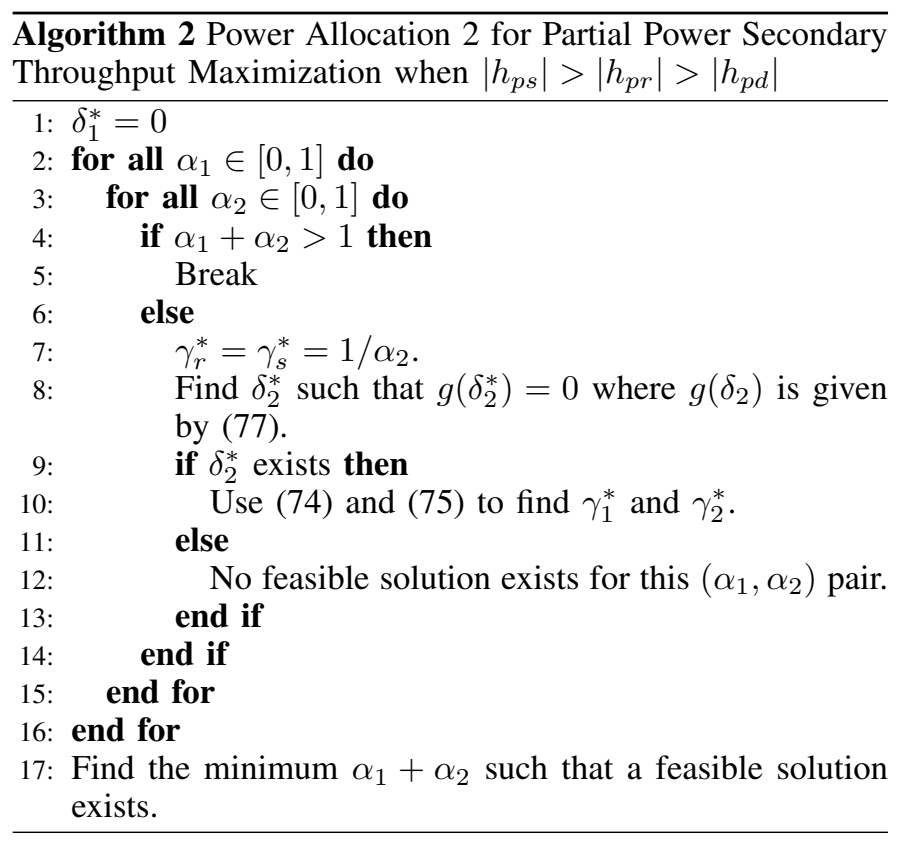

where $h_{s}$ is the channel gain between the secondary source and its receiver while $\delta_{s}=\frac{1-\alpha_{2}\left(\gamma_{s}+\mu_{s}\right)}{1-\alpha_{1}-\alpha_{2}}$ is the fraction of secondary source power remaining for the third (cognitive) communication phase. By the formulation above, we account for both the time allocated for secondary communication and the power used to maintain this communication. Similarly to the previous problem, we lowerbound the solution according to the following proposition.

Proposition 3 : The primary's transmitter splits its message according to the channel conditions as follows:

1) If $\left|h_{p s}\right|>\left|h_{p r}\right|>\left|h_{p d}\right|, P$ splits its message into two parts, $m_{d}$ and $m_{r}$, as summarized in Algorithm 3.

2) If $\left|h_{p s}\right|>\left|h_{p d}\right|>\left|h_{p r}\right|$, P splits its message into $m_{d}$ and $m_{s}$ only and uses Algorithm 4.

3) If $\left|h_{p d}\right|>\left|h_{p s}\right|>\left|h_{p r}\right|$ or $\left|h_{p d}\right|>\left|h_{p r}\right|>\left|h_{p s}\right|$, P sends only $m_{d}$ through the direct communication link and the 
cognitive user's throughput is zero.

4) If $\left|h_{p r}\right|>\left|h_{p s}\right|>\left|h_{p d}\right|, R$ sends $\left(m_{s}, m_{r}\right)$ while $S$ sends $m_{s}$ only as summarized in Algorithm 5.

5) The case of $\left|h_{p r}\right|>\left|h_{p d}\right|>\left|h_{p s}\right|$ corresponds to case 2 above with the roles of $S$ and $R$ reversed so that $R$ sends $m_{r}$ and $S$ is silent.

Proof: See Appendix C.
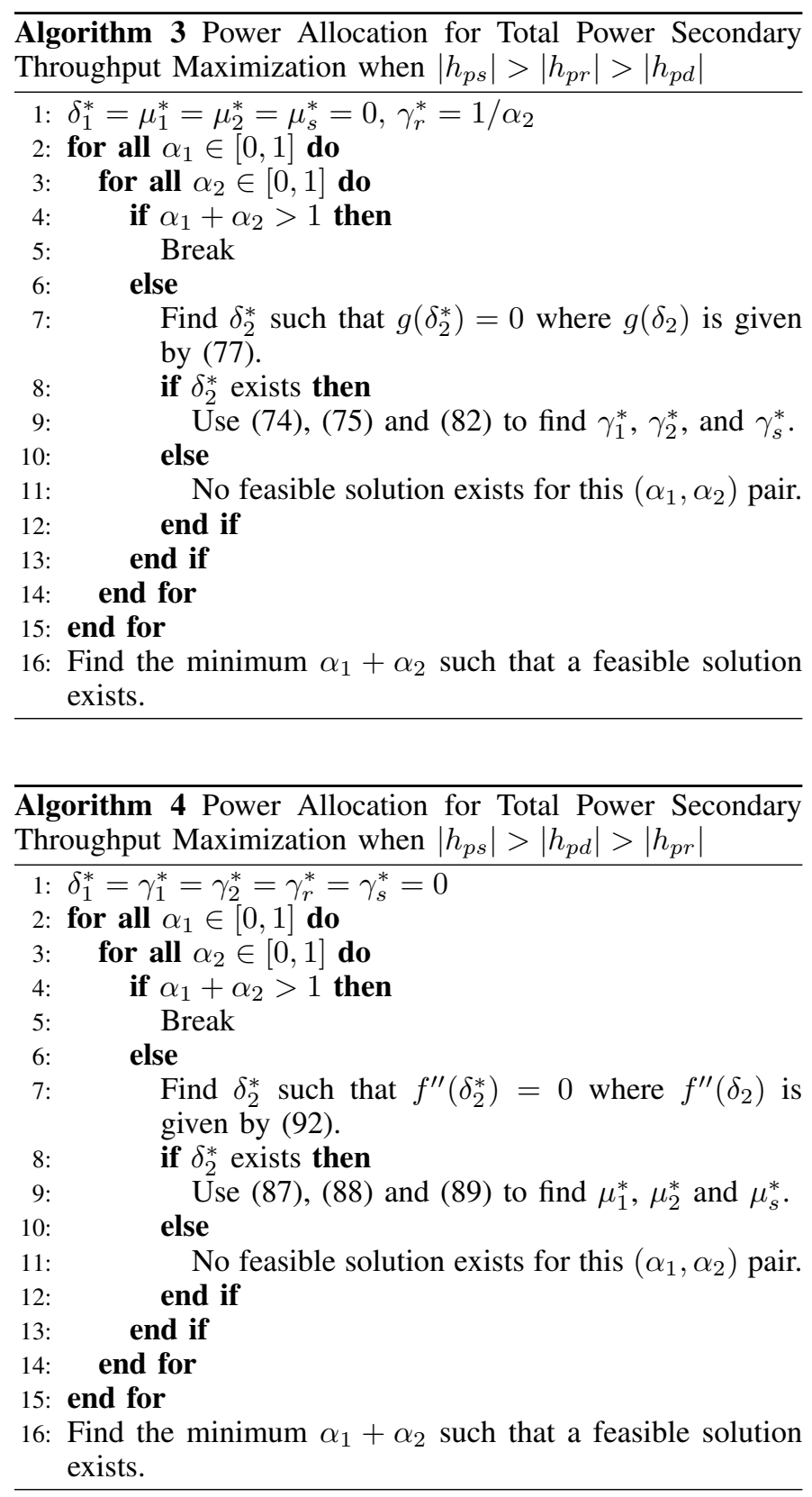

Based on the solution of the optimization problem, we note that for the first transmission case, i.e. when $\left|h_{p s}\right|>\left|h_{p r}\right|>$ $\left|h_{p d}\right|$, we have the transmission phases as follows:

- In phase 1 , node $\mathrm{P}$ sends $m_{r}$ only with power $\gamma_{1}^{*} P_{p}$. Node R decodes $\tilde{m}_{r}$ and node $\mathrm{S}$ decodes $\hat{m}_{r}$.

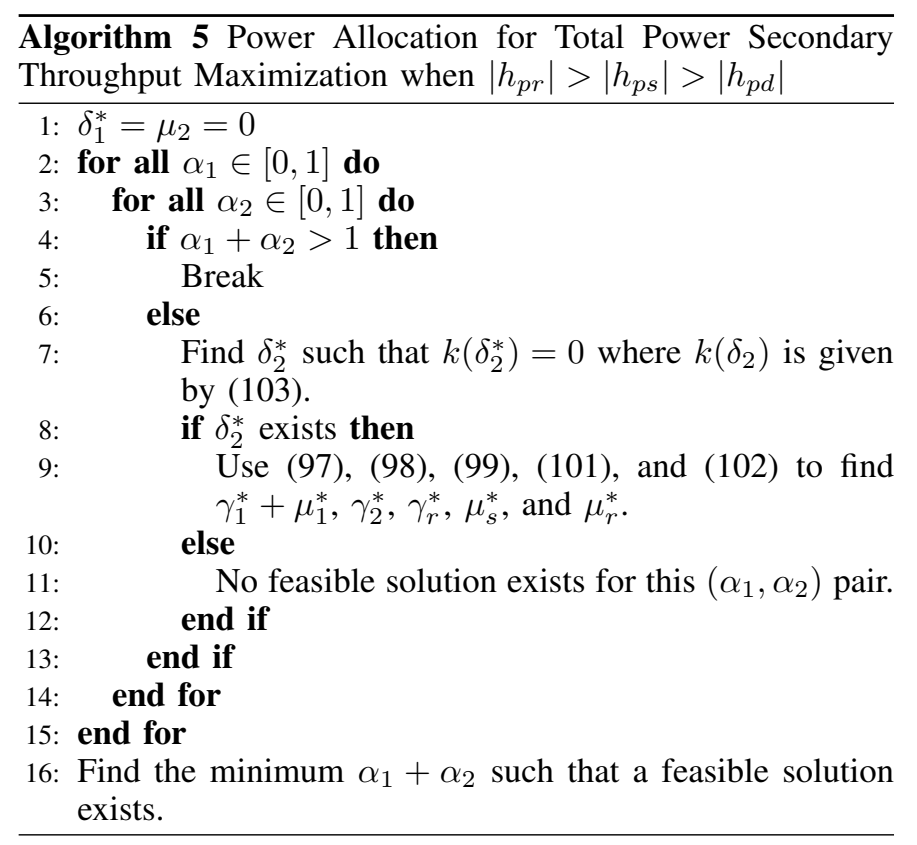

- In phase 2 , node $\mathrm{P}$ sends $m_{d}$ and $m_{r}$ only with powers $\delta_{2}^{*} P_{p}$ and $\gamma_{2}^{*} P_{p}$, respectively, while node $\mathrm{R}$ sends $\tilde{m}_{r}$ with power $\gamma_{r}^{*} P_{r}$ and node $\mathrm{S}$ sends $\hat{m}_{r}$ with power $\gamma_{s}^{*} P_{s}$.

\section{NumericAl RESUlts}

In this section, we show the performance of the two proposed schemes under the partial and total power budgets and compare our approach with various reference schemes. To the best of our knowledge, no schemes which employ the same access model that we proposed exist. Nevertheless, some of the existing works in the literature can be modified for a fair comparison. We begin with the solution to the partial power budget problem and then proceed to the total power one.

For the optimization problem involving the partial power budget constraint, we compare our algorithm with best relay selection [22]. For this comparison, we only consider case 1 of proposition 2, i.e. $\left|h_{p s}\right|>\left|h_{p r}\right|>\left|h_{p d}\right|{ }^{4}$ For best relay selection, the primary picks the node that has the best end-to-end signal-to-noise ratio (SNR) to relay its message. As clear from the simulation parameters shown in Fig. 2, the primary selects $R$ as a relaying node in this instance since $\min \left(\left|h_{p r}\right|^{2} P_{p},\left|h_{r d}\right|^{2} P_{r}^{p}\right)>\min \left(\left|h_{p s}\right|^{2} P_{p},\left|h_{s d}\right|^{2} P_{s}^{p}\right)$. We can recover two rate constraints for such scheme by setting $\mu_{1}=\mu_{2}=\mu_{s}=\gamma_{s}=0$ in (19), (20) and (21). We then solve the throughput optimization problem using a similar approach to the one described in Section III.

Fig. 2 shows the attainable secondary throughput using Algorithm 1 and best relay selection. It is clear from Fig. 2 that it is suboptimal to rely only on one link since significant throughput loss can occur.

We note that the attainable primary rate is higher than the direct-link rate $R_{d l}=0.585$. This happens since, through

\footnotetext{
${ }^{4}$ In the next subsection, we will also consider the throughput averaged over all the possible communication cases.
} 


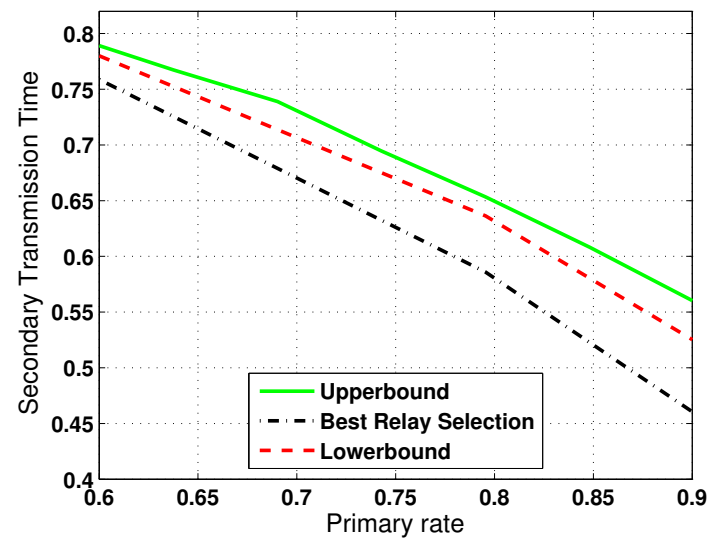

Fig. 2. Comparison between the proposed algorithm lowerbound and upperbound solutions and best relay selection. The channel parameters are chosen as follows: $\left|h_{p d}\right|^{2}=0.5,\left|h_{p r}\right|^{2}=1.5,\left|h_{p s}\right|^{2}=1.7,\left|h_{r d}\right|^{2}=1.2$ and $\left|h_{s d}\right|^{2}=1.6$. The powers are $P_{p}=1, P_{s}^{p}=0.5$ and $P_{r}^{p}=2$, and the noise variance $N=1$. Based on the transmit power and the channel gains of the primary network, the direct-link rate $R_{d l}=0.585$.

cooperative relaying, we can garner a much higher rate for $\mathrm{P}$ than what is possible through the direct link alone while still achieving a non-zero secondary throughput. For further insights, in Fig. 3, we plot the optimized values of $\alpha_{1}$ and $\alpha_{2}{ }^{5}$. It is noted that the most significant gains in throughput for the cognitive network come from minimizing $\alpha_{2}$, since this is the transmission phase where the cognitive nodes assist primary communication and significant reductions in time duration are possible in this phase.

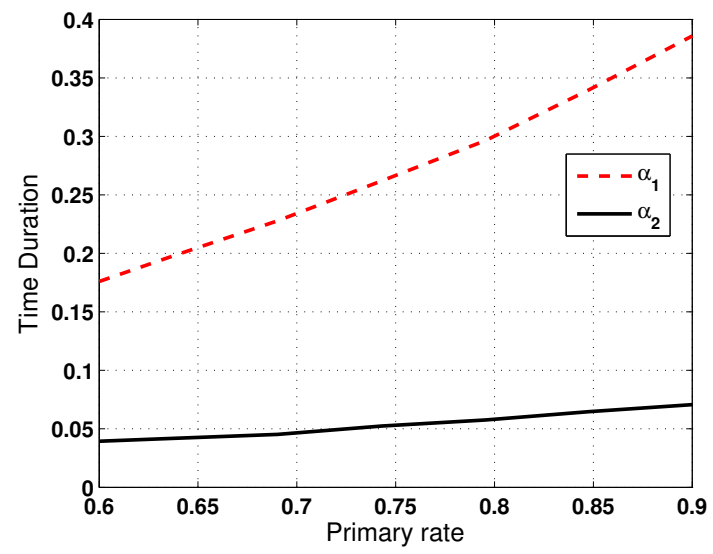

Fig. 3. Variation of $\alpha_{1}$ and $\alpha_{2}$ as a function of the primary rate. The channel parameters are: $\left|h_{p d}\right|^{2}=0.5,\left|h_{p r}\right|^{2}=1.5,\left|h_{p s}\right|^{2}=1.7,\left|h_{r d}\right|^{2}=1.2$ and $\left|h_{s d}\right|^{2}=1.6$. The powers are $P_{p}=1, P_{s}^{p}=0.5$ and $P_{r}^{p}=2$, and the noise variance $N=1$. Based on the transmit power and the channel gains of the primary network, the direct-link rate $R_{d l}=0.585$.

Now, for the case of the optimization problem with the total

\footnotetext{
${ }^{5}$ Herein, we averaged the $\alpha_{1}$ and $\alpha_{2}$ values attained from the upperbound and lowerbound solutions.
}

power budget constraint, we attempt to compare our work with two reference schemes that are detailed in the next subsection.

\section{A. Reference Schemes}

Herein, we start by comparing our approach with a dualhop scheme which does not utilize the direct link and uses one relay for transmission as described in [17]. The communication scheme can be summarized as follows: A fraction, $W_{1}$, of the primary bandwidth is allocated to primary communication, while the remaining spectrum is allocated for cognitive operation. For the secondary user to gain access to this spectrum, it must assist the primary communication in its $W_{1}$ share of the bandwidth. This assistance is facilitated by dividing the primary time slot into two equal phases such that during the first phase, the primary relays its message to the cognitive node, and during the second phase, the secondary node sends this data to the primary destination. The spectrum released by the primary to the secondary user is optimized by the latter's ability to meet a target rate for the primary network. If the target rate is not met, no cooperation is possible. While the authors use the direct-link rate as the metric for the minimum required primary rate, the direct link itself is not used for communication. The design objective in that work is to maximize the primary user power savings and the secondary throughput.

While amplify-and-forward is used in the above described scheme, we modified it here to DF relaying, like our scheme. Based on this, the rate equation governing this scheme can be written as: 6

$$
R_{p} \leq \frac{W_{1}}{2} \min \left\{\log _{2}\left(1+\frac{\left|h_{p s}\right|^{2} P_{p 1}}{N}\right), \log _{2}\left(1+\frac{\left|h_{s d}\right|^{2} P_{s 1}}{N}\right)\right\},
$$

where $P_{p 1}$ is the power used by the primary over the first transmission phase in the $W_{1}$ bandwidth, i.e. $\frac{W_{1}}{2} P_{p 1}=P_{p}$, while $P_{s 1}$ is the fraction of secondary power dedicated to assisting the primary, i.e.

$$
\frac{W_{1}}{2} P_{s 1}+\left(1-W_{1}\right) P_{s 2}=P_{s},
$$

where $P_{s 2}$ is the power used by the secondary for its own operation in the $\left(1-W_{1}\right)$ bandwidth. It is noted that $R_{p} \geq R_{d l}$ should also be satisfied to provide an incentive for the primary to cooperate. In order for $R_{p}$ to be maximized in (24), we must set $\left|h_{p s}\right|^{2} P_{p 1}=\left|h_{s d}\right|^{2} P_{s 1}$. Using the previous relations, we are able to obtain the optimal power and bandwidth allocations to allow secondary access.

Fig. 4 shows the resulting secondary throughput with the variation in the required primary rate. For a fair comparison with the dual-hop scheme, since it involves only one relay, we ran our simulations giving this relay the power of the two relays in our model. The results have been averaged over 2000 simulation runs of the Rayleigh distributed channel gains. The superiority of our scheme is clear and we attribute this to a number of key differences from this work's model: i) We utilize the direct link for communication, ii) We utilize

\footnotetext{
${ }^{6}$ Assume here only $S$ is the node available for relaying.
} 
superposition coding which has been known to improve the region of achievable rates for collaborative wireless users, and iii) We optimize the duration of the primary user transmission phases, which can garner significant throughput gains for the cognitive user without degrading primary performance.

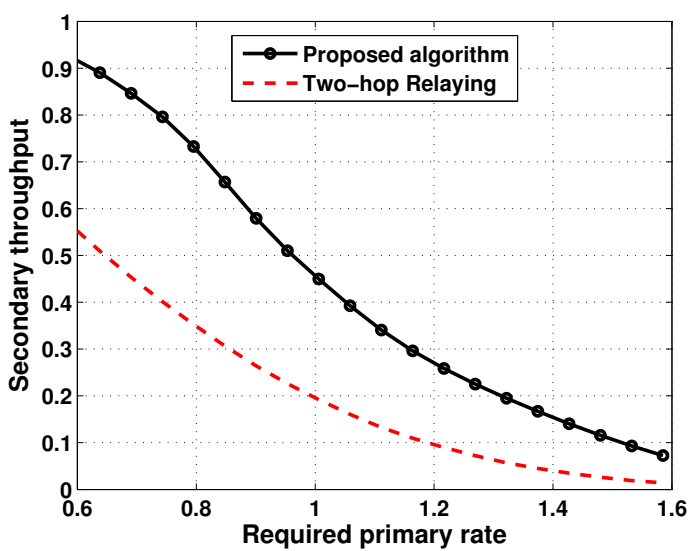

Fig. 4. Comparison between the proposed algorithm and dual-hop relaying for the optimization problem involving a total power constraint. The system parameters are the same as in Fig. 2 except that the instantaneous values of the channel gains in Fig. 2 are taken as the averages for the simulations.

The second reference scheme takes a different approach to the cognitive access problem. In [23], an interference channel model is assumed between the primary and secondary transmitter-receiver pair, i.e. the sum rate for the primary and secondary users can be written as,

$$
\begin{aligned}
R\left(P_{s}^{\prime}, P_{p}^{\prime}\right)=\log _{2} & \left(1+\frac{P_{p}^{\prime}\left|h_{p d}\right|^{2}}{N+P_{s}^{\prime}\left|h_{s d}\right|^{2}}\right) \\
+ & \log _{2}\left(1+\frac{P_{s}^{\prime}\left|h_{s}\right|^{2}}{N+P_{p}^{\prime}\left|h_{p r}\right|^{2}}\right),
\end{aligned}
$$

where $P_{p}^{\prime} \leq P_{p}$ and $P_{s}^{\prime} \leq P_{s}^{t}$ are fractions of the total primary and secondary powers, respectively. ${ }^{7}$ For fair comparison, we make the power available to the single relay in the reference scheme $P_{s}^{t}=P_{s}+P_{r}$. Also, $h_{p r}$ and $h_{s d}$ become now interference channels caused by the primary transmitter on the secondary receiver and by the secondary transmitter on the primary receiver, respectively. The authors solve the following optimization problem:

$$
\begin{aligned}
\left(P_{s}^{\prime *}, P_{p}^{\prime *}\right)= & \max _{P_{s}^{\prime}, P_{p}^{\prime}} R\left(P_{s}^{\prime}, P_{p}^{\prime}\right) \\
\text { s.t. } \quad & 0 \leq P_{s}^{\prime} \leq P_{s}^{t} \\
& 0 \leq P_{p}^{\prime} \leq P_{p} \\
& \log _{2}\left(1+\frac{P_{p}^{\prime}\left|h_{p d}\right|^{2}}{N+P_{s}^{\prime}\left|h_{s d}\right|^{2}}\right) \geq R_{\text {min }}
\end{aligned}
$$

\footnotetext{
${ }^{7}$ In that work, the primary and secondary nodes may opt not to use their full available power for communication. For convenience, we have also used a different notation for the total power of the single relay in the reference scheme as it involves the sum power of the two relays in our scheme.
}

where $R_{\min }$ is the minimum required primary rate constrained as

$$
R_{\text {min }} \leq \log _{2}\left(1+\frac{P_{p}^{\prime}\left|h_{p d}\right|^{2}}{N}\right) \leq R\left(0, P_{p}\right),
$$

Under this interference model, the maximum achievable primary rate is the direct-link rate with the maximum primary power. Hence the upperbound in (28) is $R\left(0, P_{p}\right)=R_{d l}$. The authors show that the maximum sum rate occurs on one of the points that belong to the set

$$
S_{c}=\left\{\left(0, P_{p}\right),\left(P_{s}^{t}, P_{p}\right),\left(P_{s}^{t}, \eta P_{s}^{t}+\beta\right)\right\},
$$

if $\eta P_{s}^{t}+\beta \leq P_{p}$, otherwise

$$
S_{c}=\left\{\left(0, P_{p}\right),\left(\frac{P_{p}-\beta}{\eta}, P_{p}\right)\right\},
$$

where $\eta=\left(2^{R_{\min }}-1\right)\left|h_{s d}\right|^{2} /\left|h_{p d}\right|^{2} \quad$ while $\beta=$ $\left(2^{R_{\min }}-1\right) N /\left|h_{p d}\right|^{2}$.

In Fig. 5, we plot the resulting sum rate against the variation in the direct-link channel gain using our approach and the solution suggested in [23]. The first breakpoint of throughput in the proposed algorithm comes from the fact that case 2 of Proposition 3 applies once $\left|h_{p d}\right|>\left|h_{p r}\right|$. This necessarily degrades throughput since only message $m_{s}$ is sent from node $\mathrm{S}$ and no message is sent from node R. For our approach, cooperation becomes impossible beyond $\left|h_{p d}\right|>\left|h_{p s}\right|$ where the second break point occurs at $\left|h_{p s}\right|^{2}=2$, and the only achievable rate is the direct-link one. As for the interference model, we purposefully set $\left|h_{s d}\right|^{2}$ to a small value, 0.5 , to mimic a small interference case in addition to making the primary rate requirement low, only $0.5 R_{d l}$. In that case, we can see that beyond $\left|h_{p d}\right|>\left|h_{p s}\right|$, the interference model can outperform our scheme. However, under this same small interference value but with a larger rate requirement $R_{p}=$ $R_{\text {min }}=1.1 R_{d l}$, the interference model can only garner the direct-link rate while our approach can get more than the direct-link rate, in addition to a non-zero throughput for the secondary. This is also clear in Fig. 6, where $\left|h_{s d}\right|^{2}=0.5$, the rate requirement is small, however $\left|h_{p s}\right|^{2}=5$. Under such circumstances, the advantages of our scheme are clear as more throughput is possible through our cooperative model. ${ }^{8}$

\section{CONCLUSION}

We considered cooperative communication in a spectrumsharing network through a three-message superposition coding scheme. The scheme involves dividing the time resource into three phases. The first two phases are used for primary communications while the third phase is used for cognitive operation. Secondary nodes aim to maximize their throughput while guaranteeing a minimum rate for the primary user. We formulated two optimization problems that maximize the aforementioned objective subject to information-theoretic rate constraints and partial/total power constraints. The optimization variables are

\footnotetext{
${ }^{8}$ If the primary rate requirement is above the direct-link rate, it is not clear what incentives the primary user would have to participate in the model proposed in [23].
} 


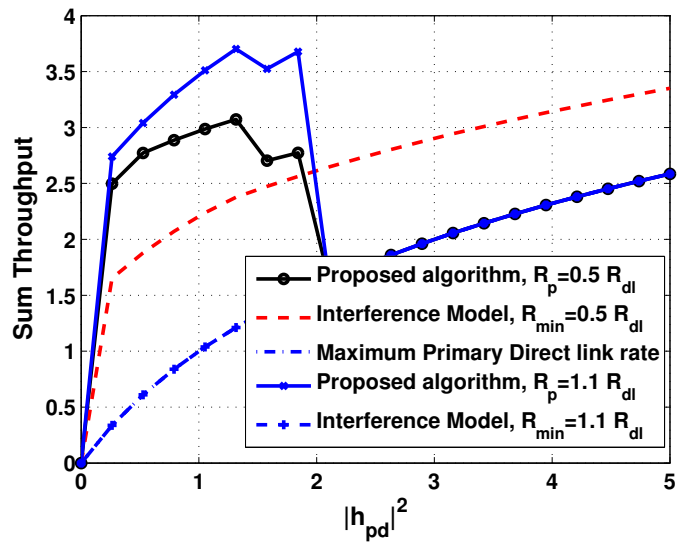

Fig. 5. Comparison between the proposed algorithm and the interference model of [23] for the problem involving a total power constraint. We choose $\left|h_{p r}\right|^{2}=1.5,\left|h_{p s}\right|^{2}=2,\left|h_{r d}\right|^{2}=1.2$ and $\left|h_{s d}\right|^{2}=0.5$. The powers are $P_{p}=1, P_{s}=0.5, P_{r}=2$ and $P_{s}^{t}=2.5$, and the noise variance $N=1$.

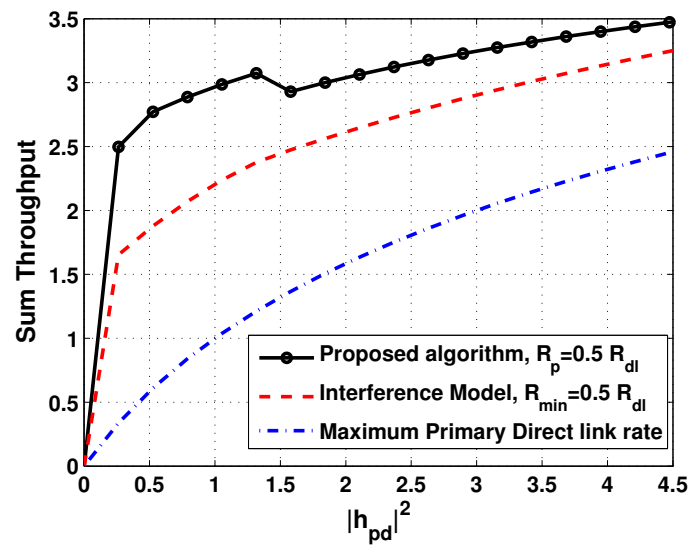

Fig. 6. Comparison between proposed algorithm and dual-hop relaying for the problem involving a total power constraint. We choose: $\left|h_{p r}\right|^{2}=1.5$, $\left|h_{p s}\right|^{2}=5,\left|h_{r d}\right|^{2}=1.2$ and $\left|h_{s d}\right|^{2}=0.5$. The powers are $P_{p}=1$, $P_{s}=0.5, P_{r}=2$ and $P_{s}^{t}=2.5$, and the noise variance $N=1$.

the time durations of the transmission phases and power allocations to the different messages. Because the two problems are mathematically intractable to solve, we provided upperbound and lowerbound analytical solutions to our optimization problem and showed that significant throughput gains can be achieved for all network users even when considering the lowerbound solution only. We also demonstrated the advantage of our proposed scheme compared to a number of reference schemes that include best relay selection, dual-hop routing and an interference channel model.

\section{APPENDIX A}

\section{ACHiEvable Rates for Suggested COMMUnication SCHEME}

Without loss of generality, assume that $\left(m_{d}, m_{r}, m_{s}\right)=$ $(1,1,1)$ was sent. Then, at the secondary receiver, error will happen under two events:

$$
\begin{gathered}
E_{r 1}=\left\{\left(Y_{r}^{\alpha_{1} n}, U^{\alpha_{1} n}(1) \notin \mathcal{A}_{\epsilon}^{\alpha_{1} n}\right\},\right. \\
E_{r 2}=\left\{\left(Y_{r}^{\alpha_{1} n}, U^{\alpha_{1} n}\left(m_{r}\right)\right) \in \mathcal{A}_{\epsilon}^{\alpha_{1} n}\right\},
\end{gathered}
$$

It can be shown that $E_{r 1} \rightarrow 0$ as $n \rightarrow \infty$ by the asymptotic equipartition property. If we analyze the event $E_{r 2}$, we get

$$
p\left(E_{r 2}\right)=\sum_{i=1}^{2^{n R_{r}}-1} p\left(E_{r 2, i}\right),
$$

where

$$
\begin{aligned}
p\left(E_{r 2, i}\right) & \leq \sum_{\left(Y_{r}, U\right) \in \mathcal{A}_{\epsilon}^{\alpha_{1} n}} p(U) p\left(Y_{r}\right) \\
& \leq 2^{\alpha_{1} n\left(H\left(Y_{r}, U\right)+\epsilon\right)} 2^{-\alpha_{1} n(H(U)-\epsilon)} 2^{-\alpha_{1} n\left(H\left(Y_{r}\right)-\epsilon\right)} \\
& \leq 2^{-\alpha_{1} n\left(I\left(Y_{r}, U\right)-3 \epsilon\right)}
\end{aligned}
$$

Therefore, $p\left(E_{r 2}\right) \leq 2^{n R_{r}-\alpha_{1} n\left(I\left(Y_{r}, U\right)-3 \epsilon\right)}$, so that $p\left(E_{r 2}\right) \rightarrow$ 0 only if $R_{r} \leq \alpha_{1} I\left(Y_{r}, U\right)$.

Similarly, at the secondary source, error will happen if $m_{r}$ is decoded erroneously for any $m_{s}$ if

$$
\left\{Y_{s}^{\alpha_{1} n}, L^{\alpha_{1} n}\left(m_{s}\right), U^{\alpha_{1} n}\left(m_{r}\right)\right\} \in \mathcal{A}_{\epsilon}^{\alpha_{1} n} .
$$

Error will also happen at the secondary source if $m_{s}$ is decoded erroneously, given that $m_{r}$ is correct, i.e.

$$
\left\{Y_{s}^{\alpha_{1} n}, L^{\alpha_{1} n}\left(m_{s}\right), U^{\alpha_{1} n}(1)\right\} \in \mathcal{A}_{\epsilon}^{\alpha_{1} n} .
$$

Following the same procedure as (33) and (34) will yield the constraints

$$
\begin{aligned}
R_{r}+R_{s} & \leq \alpha_{1} I\left(Y_{s} ; U, L\right), \\
R_{s} & \leq \alpha_{1} I\left(Y_{s} ; L \mid U\right) .
\end{aligned}
$$

Now, at the destination, if $m_{r}$ is decoded erroneously for any $m_{s}$ and $m_{d}$, the error event is $E_{r}=E_{r a} \cap E_{r b}$, where,

$$
\begin{aligned}
E_{r a}= & \left\{Y_{1}^{\alpha_{1} n}, L^{\alpha_{1} n}\left(m_{s}\right), U^{\alpha_{1} n}\left(m_{r}\right), X_{1}^{\alpha_{1} n}\left(m_{d}, m_{r}, m_{s}\right)\right\} \in \mathcal{A}_{\epsilon}^{\alpha_{1} n}, \\
E_{r b}= & \left\{Y_{2}^{\alpha_{2} n}, L^{\alpha_{2} n}\left(m_{s}\right), U^{\alpha_{2} n}\left(m_{r}\right), X_{r}^{\alpha_{2} n}\left(m_{r}\right), X_{s}^{\alpha_{2} n}\left(m_{r}, m_{s}\right),\right. \\
& \left.X_{2}^{\alpha_{2} n}\left(m_{d}, m_{r}, m_{s}\right)\right\} \in \mathcal{A}_{\epsilon}^{\alpha_{2} n} .
\end{aligned}
$$

However, if $m_{s}$ is in error, given that $m_{r}$ is correct, and for any $m_{d}$, the error event is $E_{s}=E_{s a} \cap E_{s b}$, where

$$
\begin{aligned}
E_{s a}= & \left\{Y_{1}^{\alpha_{1} n}, L^{\alpha_{1} n}\left(m_{s}\right), U^{\alpha_{1} n}(1), X_{1}^{\alpha_{1} n}\left(m_{d}, 1, m_{s}\right)\right\} \in \mathcal{A}_{\epsilon}^{\alpha_{1} n}, \\
E_{s b}= & \left\{Y_{2}^{\alpha_{2} n}, L^{\alpha_{2} n}\left(m_{s}\right), U^{\alpha_{2} n}(1), X_{r}^{\alpha_{2} n}(1), X_{s}^{\alpha_{2} n}\left(1, m_{s}\right),\right. \\
& \left.X_{2}^{\alpha_{2} n}\left(m_{d}, 1, m_{s}\right)\right\} \in \mathcal{A}_{\epsilon}^{\alpha_{2} n} .
\end{aligned}
$$

Finally, if $m_{d}$ is in error, given that $m_{r}$ and $m_{s}$ are correct, the error is $E_{d}=E_{d a} \cap E_{d b}$, where

$$
\begin{aligned}
E_{d a}= & \left\{Y_{1}^{\alpha_{1} n}, L^{\alpha_{1} n}(1), U^{\alpha_{1} n}(1), X_{1}^{\alpha_{1} n}\left(m_{d}, 1,1\right)\right\} \in \mathcal{A}_{\epsilon}^{\alpha_{1} n}, \\
E_{d b}= & \left\{Y_{2}^{\alpha_{2} n}, L^{\alpha_{2} n}(1), U^{\alpha_{2} n}(1), X_{r}^{\alpha_{2} n}(1), X_{s}^{\alpha_{2} n}(1,1),\right. \\
& \left.X_{2}^{\alpha_{2} n}\left(m_{d}, 1,1\right)\right\} \in \mathcal{A}_{\epsilon}^{\alpha_{2} n} .
\end{aligned}
$$


These three error events will yield the following inequalities,

$$
\begin{aligned}
R_{d}+R_{r}+R_{s} & \leq \alpha_{1} I\left(Y_{1} ; U, L, X\right)+\alpha_{2} I\left(Y_{2} ; U, L, X, X_{r}, X_{s}\right), \\
R_{d}+R_{s} & \leq \alpha_{1} I\left(Y_{1} ; L, X \mid U\right)+\alpha_{2} I\left(Y_{2} ; L, X, X_{s} \mid U, X_{r}\right), \\
R_{d} & \leq \alpha_{1} I\left(Y_{1} ; X \mid U, L\right)+\alpha_{2} I\left(Y_{2} ; X \mid U, L, X_{r}, X_{s}\right) .
\end{aligned}
$$

Finally, by applying the Fourier-Motzkin elimination method to the above inequalities, we get the inequalities in (5)-(9).

\section{APPENDIX B \\ Throughrut Maximization With Partial Power BUDGET}

Considering the rate constraints of (19), (20) and (21), it can be verified that the problem is highly non-linear in the optimization variables so that an analytically tractable solution remains elusive. Hence, we consider a simple modification to these constraints that will help us lowerbound and upperbound the solution while yielding closed-form expressions for the optimization variables. We start with the case of $\left|h_{p s}\right|>$ $\left|h_{p r}\right|>\left|h_{p d}\right|$. For the first lowerbound, consider a modification of the inequality (19) to be,

$$
\begin{aligned}
R_{p} \leq & \alpha_{1} \log _{2}\left(1+\frac{\left|h_{p r}\right|^{2}\left(\gamma_{1}+\mu_{1}\right) P_{p}}{N+\left|h_{p r}\right|^{2} P_{p} \delta_{1}}\right)+\alpha_{1} \log _{2}\left(1+\frac{\left|h_{p d}\right|^{2} \delta_{1} P_{p}}{N}\right) \\
& +\alpha_{2} \log _{2}\left(1+\frac{\left|h_{p d}\right|^{2} \delta_{2} P_{p}}{N}\right)=\underline{I}_{1}<I_{1} .
\end{aligned}
$$

\section{Comparing}

show that

$$
\text { (19) with }
$$

(42),

can $\alpha_{1} \log _{2}\left(1+\frac{\left|h_{p r}\right|^{2} \gamma_{1} P_{p}}{N+\left|h_{p r}\right|^{2} P_{p}\left(\delta_{1}+\mu_{1}\right)}\right)+\alpha_{1} \log _{2}\left(1+\frac{\left|h_{p s}\right|^{2} \mu_{1} P_{p}}{N+\left|h_{p s}\right|^{2} P_{p} \delta_{1}}\right)$ so that $\underline{I}_{1}$ is tighter than $I_{1}$. If we now solve the modified problem

$$
\begin{array}{cl}
\min _{\omega} & \alpha_{1}+\alpha_{2}, \\
\text { s.t. } & \underline{I}_{1} \geq R_{p}, \quad I_{2} \geq R_{p}, \quad I_{3} \geq R_{p}, \\
& R_{p} \geq \log _{2}\left(1+\frac{\left|h_{p d}\right|^{2} P_{p}}{N}\right)=R_{d l}, \\
& \alpha_{1}\left(\delta_{1}+\gamma_{1}+\mu_{1}\right)+\alpha_{2}\left(\delta_{2}+\gamma_{2}+\mu_{2}\right) \leq 1, \\
& \alpha_{2} \gamma_{r} P_{r}^{p} \leq P_{r}^{p}, \alpha_{2}\left(\mu_{s}+\gamma_{s}\right) P_{s}^{p} \leq P_{s}^{p},
\end{array}
$$

a solution to the problem (43), if it exists, will serve as lowerbound to the original problem of (22). As a function of both $\alpha_{1}, \alpha_{2}$ and the other variables of $\omega$, this modified optimization problem is still analytically intractable to approach unless a two-dimensional search is carried out over $\alpha_{1}$ and $\alpha_{2}$. In this case, for the given $\alpha_{1}$ and $\alpha_{2}$ values, the objective becomes a constant, which transforms our problem into finding a feasible solution that obeys all constraints. Hence, we write the Lagrangian as:

$$
\mathcal{L}(\omega)=-\sum_{i=1}^{15} \lambda_{i} C_{i}(\omega),
$$

where $C_{1}(\omega)=\underline{I}_{1}-R_{p}, C_{2}(\omega)=I_{2}-R_{p}, C_{3}(\omega)=I_{3}-R_{p}$, $C_{4}(\omega)=1-\alpha_{1}\left(\delta_{1}+\gamma_{1}+\mu_{1}\right)-\alpha_{2}\left(\delta_{2}+\gamma_{2}+\mu_{2}\right), C_{5}(\omega)=$ $1-\alpha_{2} \gamma_{r}, C_{6}(\omega)=1-\alpha_{2}\left(\mu_{s}+\gamma_{s}\right), C_{7}(\omega)=\delta_{1}, C_{8}(\omega)=\mu_{1}$, $C_{9}(\omega)=\gamma_{1}, C_{10}(\omega)=\delta_{2}, C_{11}(\omega)=\mu_{2}, C_{12}(\omega)=\gamma_{2}$,
$C_{13}(\omega)=\gamma_{s}, C_{14}(\omega)=\mu_{s}$ and $C_{15}(\omega)=\gamma_{r} .{ }^{9}$ The KarushKuhn-Tucker (KKT) conditions are:

$$
\begin{aligned}
& \nabla_{\omega} \mathcal{L}\left(\omega^{*}, \lambda^{*}\right)=0, \\
& C_{i}\left(\omega^{*}\right) \geq 0, \quad \lambda_{i}^{*} \geq 0, \quad \lambda_{i}^{*} C_{i}\left(\omega^{*}\right)=0 .
\end{aligned}
$$

Differentiating the Lagrangian, we get:

$$
\begin{aligned}
\frac{\partial \mathcal{L}}{\partial \delta_{1}} & =-\frac{\lambda_{1} g_{p d}}{N+g_{p d} \delta_{1} P_{p}}-\frac{\lambda_{2} g_{p d}}{N+g_{p d}\left(\delta_{1}+\mu_{1}+\gamma_{1}\right) P_{p}} \\
& +\frac{\lambda_{1} g_{p r}^{2} P_{p}\left(\gamma_{1}+\mu_{1}\right)}{\left(N+g_{p r} P_{p}\left(\delta_{1}+\mu_{1}+\gamma_{1}\right)\right)\left(N+g_{p r} P_{p} \delta_{1}\right)} \\
& +\frac{\lambda_{3} g_{p r}^{2} P_{p} \gamma_{1}}{\left(N+g_{p r} P_{p}\left(\delta_{1}+\mu_{1}+\gamma_{1}\right)\right)\left(N+g_{p r} P_{p}\left(\delta_{1}+\mu_{1}\right)\right)} \\
& -\frac{\lambda_{3} g_{p d}}{N+g_{p d}\left(\delta_{1}+\mu_{1}\right) P_{p}}+\lambda_{4} \frac{\ln 2}{P_{p}}-\lambda_{7} \frac{\ln 2}{\alpha_{1} P_{p}}=0 \\
\frac{\partial \mathcal{L}}{\partial \mu_{1}} & =-\frac{\lambda_{1} g_{p r}}{N+g_{p r}\left(\delta_{1}+\gamma_{1}+\mu_{1}\right) P_{p}}-\frac{\lambda_{2} g_{p d}}{N+g_{p d}\left(\delta_{1}+\mu_{1}+\gamma_{1}\right) P_{p}} \\
& +\frac{\lambda_{p}\left(\gamma_{1}\right)}{\left(N+g_{p r} P_{p}\left(\delta_{1}+\mu_{1}+\gamma_{1}\right)\right)\left(N+g_{p r} P_{p}\left(\delta_{1}+\mu_{1}\right)\right)} \\
& -\frac{\lambda_{3} g_{p d}}{N+g_{p d}\left(\delta_{1}+\mu_{1}\right) P_{p}}+\lambda_{4} \frac{\ln 2}{P_{p}}-\lambda_{8} \frac{\ln 2}{\alpha_{1} P_{p}}=0 \\
\frac{\partial \mathcal{L}}{\partial \gamma_{1}}= & -\frac{\lambda_{1} g_{p r}}{N+g_{p r}\left(\delta_{1}+\gamma_{1}+\mu_{1}\right) P_{p}}-\frac{\lambda_{2} g_{p d}}{N+g_{p d}\left(\delta_{1}+\mu_{1}+\gamma_{1}\right) P_{p}} \\
- & \frac{\lambda_{3} g_{p r}}{N+g_{p r}\left(\delta_{1}+\gamma_{1}+\mu_{1}\right) P_{p}}+\lambda_{4} \frac{\ln 2}{P_{p}}-\lambda_{9} \frac{\ln 2}{\alpha_{1} P_{p}}=0
\end{aligned}
$$

where $g_{i j}=\left|h_{i j}\right|^{2}$ above. We note that for the proposed scheme to be superior to direct transmission and given the assumption $g_{p s}>g_{p r}>g_{p d}, \gamma_{1}$ and $\mu_{1}$ must be set to nonzero values. Hence, by the KKT conditions, $\lambda_{8}=\lambda_{9}=0$. Also, since $\gamma_{1}>0$ and $\mu_{1}>0$, we must have $\gamma_{r}>0, \mu_{s}>0$ and $\gamma_{s}>0$, therefore $\lambda_{13}=\lambda_{14}=\lambda_{15}=0$. Also note that (47)-(48), gives:

$$
\lambda_{3}\left(\frac{g_{p r}}{N+g_{p r} P_{P}\left(\delta_{1}+\mu_{1}\right)}-\frac{g_{p d}}{N+g_{p d} P_{p}\left(\delta_{1}+\mu_{1}\right)}\right)=0,
$$

and since $g_{p r}>g_{p d}$, then $\lambda_{3}=0$. The remaining KKT conditions are

$$
\begin{aligned}
\frac{\partial \mathcal{L}}{\partial \delta_{2}} & =-\frac{\lambda_{1} g_{p d}}{N+g_{p d} P_{p} \delta_{2}}-\frac{\lambda_{2} g_{p d}}{\omega_{r p s} N}+\frac{\lambda_{4} \ln 2}{P_{p}}-\frac{\lambda_{10} \ln 2}{\alpha_{2} P_{p}}=0 \\
\frac{\partial \mathcal{L}}{\partial \mu_{2}} & =-\frac{\lambda_{2}}{\omega_{r p s}}\left[\frac{g_{p d} P_{p}}{N}+\sqrt{\frac{g_{s d} g_{p d} \mu_{s} P_{s}^{p} P_{p}}{\mu_{2}}}\right]+\lambda_{4} \ln 2 \\
& -\frac{\lambda_{11} \ln 2}{\alpha_{2}}=0
\end{aligned}
$$

$$
\begin{aligned}
\frac{\partial \mathcal{L}}{\partial \gamma_{2}} & =-\frac{\lambda_{2}}{\omega_{r p s}}\left[\frac{g_{p d} P_{p}}{N}+\sqrt{\frac{g_{r d} g_{p d} \gamma_{r} P_{r}^{p} P_{p}}{\gamma_{2}}}+\sqrt{\frac{g_{s d} g_{p d} \gamma_{s} P_{s}^{p} P_{p}}{\gamma_{2}}}\right] \\
& +\lambda_{4} \ln 2-\frac{\lambda_{12} \ln 2}{\alpha_{2}}=0
\end{aligned}
$$

\footnotetext{
${ }^{9}$ The constraints $C_{7}-C_{15}$ are non-negativity constraints on the optimization variables.
} 


$$
\begin{aligned}
\frac{\partial \mathcal{L}}{\partial \gamma_{r}} & =-\frac{\lambda_{2}}{\omega_{r p s}}\left[\frac{g_{r d} P_{r}^{p}}{N}+\sqrt{\frac{g_{r d} g_{p d} \gamma_{2} P_{r}^{p} P_{p}}{\gamma_{r}}}+\sqrt{\frac{g_{s d} g_{r d} \gamma_{s} P_{s}^{p} P_{r}^{p}}{\gamma_{r}}}\right] \\
& +\lambda_{5} \ln 2=0, \\
\frac{\partial \mathcal{L}}{\partial \gamma_{s}} & =-\frac{\lambda_{2}}{\omega_{r p s}}\left[\frac{g_{s d} P_{s}^{p}}{N}+\sqrt{\frac{g_{s d} g_{p d} \gamma_{2} P_{s}^{p} P_{p}}{\gamma_{s}}}+\sqrt{\frac{g_{s d} g_{r d} \gamma_{r} P_{s}^{p} P_{r}^{p}}{\gamma_{s}}}\right] \\
& +\lambda_{6} \ln 2=0, \\
\frac{\partial \mathcal{L}}{\partial \mu_{s}} & =-\frac{\lambda_{2}}{\omega_{r p s}}\left[\frac{g_{s d} P_{s}^{p}}{N}+\sqrt{\frac{g_{s d} g_{p d} \mu_{2} P_{s}^{p} P_{p}}{\mu_{s}}}\right]+\lambda_{6} \ln 2=0,
\end{aligned}
$$

where $\omega_{r p s}=1+\frac{g_{p d} P_{p}\left(\delta_{2}+\gamma_{2}+\mu_{2}\right)+g_{r d} P_{r}^{p} \gamma_{r}+g_{s d} P_{s}^{p}\left(\mu_{s}+\gamma_{s}\right)+\zeta}{N}$.

Using (54) and (55), we get

$$
\frac{\lambda_{2}}{\omega_{r p s}}\left(\sqrt{\frac{g_{p d} \gamma_{2} P_{p}}{\gamma_{s}}}+\sqrt{\frac{g_{r d} \gamma_{r} P_{r}^{p}}{\gamma_{s}}}\right)=\frac{\lambda_{2}}{\omega_{r p s}} \sqrt{\frac{g_{p d} \mu_{2} P_{p}}{\mu_{s}}} .
$$

Now, we need to check whether $\delta_{2}>0, \mu_{2}>0, \gamma_{2}>0$, i.e. $\lambda_{10}=\lambda_{11}=\lambda_{12}=0$. Note that the case of $\mu_{2}=0$ is not possible since it leads to an inconsistency in (56). Hence $\mu_{2}>0$ and then $\lambda_{12}=0$. Now, assume $\gamma_{2}>0$ and the associated Lagrange multiplier $\lambda_{12}=0$, then from (52) and (52), we get

$$
\frac{\lambda_{2}}{\omega_{r p s}}\left(\sqrt{\frac{g_{r d} \gamma_{r} P_{r}^{p}}{\gamma_{2}}}+\sqrt{\frac{g_{s d} \gamma_{s} P_{s}^{p}}{\gamma_{2}}}\right)=\frac{\lambda_{2}}{\omega_{r p s}} \sqrt{\frac{g_{s d} \mu_{s} P_{s}^{p}}{\mu_{2}}} .
$$

However, if we compare (56) and (57), we get by solving them together the inconsistent result that makes,

$$
\frac{\lambda_{2}}{\omega_{r p s}} \sqrt{\frac{g_{p d} g_{r d} P_{p} P_{r}^{p} \mu_{2} \gamma_{r}}{g_{s d} \mu_{s} P_{s}^{p}}}=-\frac{\lambda_{2}}{\omega_{r p s}} \sqrt{g_{r d} \gamma_{r} P_{r}^{p}} .
$$

We note from the above equations that $\lambda_{2}=0$ leads to $\lambda_{1}=$ $\lambda_{4}=\lambda_{5}=\lambda_{6}=0$ and the problem becomes trivial. Hence, $\lambda_{2}>0$. In this case, the only resolution to the above dilemma is that $\lambda_{12}>0$, i.e. $\gamma_{2}=0$. Note also that if $\delta_{2}>0$, then $\lambda_{10}=0$ and from (50) we get

$$
\frac{\lambda_{1} g_{p d}}{N+g_{p d} P_{p} \delta_{2}}=\frac{\lambda_{4} \ln 2}{P_{p}}-\frac{\lambda_{2} g_{p d}}{\omega_{r p s} N}=\frac{\lambda_{2}}{\omega_{r p s}} \sqrt{\frac{g_{s d} g_{p d} \mu_{s} P_{s}^{p} P_{p}}{\mu_{2}}},
$$

i.e. $\lambda_{1}>0$, hence $\delta_{1}=0$.

To summarize, we now have the equations of (20), (42), (56) and the power constraints $\alpha_{1}\left(\delta_{1}+\gamma_{1}+\mu_{1}\right)+\alpha_{2}\left(\delta_{2}+\gamma_{2}+\mu_{2}\right)=$ $1, \alpha_{2} \gamma_{r} P_{r}^{p}=P_{r}^{p}, \alpha_{2}\left(\mu_{s}+\gamma_{s}\right) P_{s}^{p}=P_{s}^{p}$, to solve for the 7 variables $\left[\begin{array}{llllll}\delta_{2} & \gamma_{1} & \mu_{1} & \mu_{2} & \gamma_{r} & \gamma_{s} \\ \mu_{s}\end{array}\right] .{ }^{10}$ As per the primary power

\footnotetext{
${ }^{10}$ Since (21) is not active, modifying (19) to (42) makes the individual values of $\gamma_{1}$ and $\mu_{1}$ irrelevant and only their sum counts.
}

constraint (18), $\delta_{2} \in\left[0,1 / \alpha_{2}\right]$. Hence, from (42), we get

$$
\gamma_{1}\left(\delta_{2}\right)+\mu_{1}\left(\delta_{2}\right)=\frac{N}{g_{p r} P_{p}}\left[2^{\frac{R_{p}-\alpha_{2} \log _{2}\left(1+\frac{g_{p d} \delta_{2} P_{p}}{N}\right)}{\alpha_{1}}}-1\right] \text {. }
$$

From (18), we obtain

$$
\mu_{2}\left(\delta_{2}\right)=\frac{1-\alpha_{1}\left(\gamma_{1}\left(\delta_{2}\right)+\mu_{1}\left(\delta_{2}\right)\right)-\alpha_{2} \delta_{2}}{\alpha_{2}} .
$$

Using (56), we get

$$
\mu_{s}\left(\delta_{2}\right)=\frac{\mu_{2}\left(\delta_{2}\right)}{\alpha_{2}\left(\eta+\mu_{2}\left(\delta_{2}\right)\right)},
$$

where $\eta=\frac{g_{r d} \gamma_{r} P_{r}^{p}}{g_{p d} P_{p}}$. Now, substituting the above three equations, along with $\gamma_{s}\left(\delta_{2}\right)=\frac{1}{\alpha_{2}}-\mu_{s}\left(\delta_{2}\right)$, into (20), we can numerically find $\delta_{2}$ such that

$$
\begin{aligned}
& f\left(\delta_{2}\right)=g_{p d} P_{p}\left(\delta_{2}+\mu_{2}\left(\delta_{2}\right)\right)+g_{s d}\left(\mu_{s}\left(\delta_{2}\right)+\gamma_{s}\left(\delta_{2}\right)\right)-\beta \\
& \quad+2 \sqrt{g_{r d} g_{s d} \gamma_{s}\left(\delta_{2}\right) \gamma_{r} P_{r}^{p} P_{s}^{p}}+2 \sqrt{g_{s d} g_{p d} \mu_{s}\left(\delta_{2}\right) \mu_{2}\left(\delta_{2}\right) P_{s}^{p} P_{p}}=0,
\end{aligned}
$$

where $\beta=2^{\frac{R_{p}-\alpha_{1} \log _{2}\left(1+\frac{g_{p d} P_{p}\left(\gamma_{1}\left(\delta_{2}\right)+\mu_{1}\left(\delta_{2}\right)\right)}{N}\right)}{\alpha_{2}}}-\frac{g_{r d} \gamma_{r} P_{r}^{p}}{N}-1$. Once $\delta_{2}$ is obtained, we have $\mu_{s}, \gamma_{2}, \mu_{2}$ and the sum $\gamma_{1}+\mu_{1}$.

We note that when $\gamma_{r}=1 / \alpha_{2}$, there may not be a numerical solution for $\delta_{2}$ in (63). In that case, we scan the value of $\gamma_{r} \in\left[0,1 / \alpha_{2}\right]$ and solve (20). If no value for $\delta_{2}$ can be found, then the solution is not feasible for the chosen value of $\alpha_{1}$ and $\alpha_{2}$ and active cooperation is not possible.

Similar to the approach above, an upperbound solution can be obtained by simply modifying $I_{1}$ to

$$
\begin{aligned}
R_{p} & \leq \alpha_{1} \log _{2}\left(1+\frac{\left|h_{p s}\right|^{2}\left(\gamma_{1}+\mu_{1}\right) P_{p}}{N+\left|h_{p s}\right|^{2} P_{p} \delta_{1}}\right) \\
& +\alpha_{1} \log _{2}\left(1+\frac{\left|h_{p d}\right|^{2} \delta_{1} P_{p}}{N}\right)+\alpha_{2} \log _{2}\left(1+\frac{\left|h_{p d}\right|^{2} \delta_{2} P_{p}}{N}\right)=\bar{I}_{1},
\end{aligned}
$$

and the solution proceeds exactly as above. We also propose another lowerbound. Consider the case of sending only $m_{r}$ from $\mathrm{S}$ and $\mathrm{R}$, i.e. $\mu_{1}=\mu_{2}=\mu_{s}=0$. Under such scenario, we solve the following optimization problem,

$$
\begin{aligned}
\min _{\omega} & \alpha_{1}+\alpha_{2}, \\
\text { s.t. } & I_{1}^{\prime} \geq R_{p}, \quad I_{2}^{\prime} \geq R_{p}, \\
& R_{p} \geq \log _{2}\left(1+\frac{g_{p d} P_{p}}{N}\right), \\
& \alpha_{1}\left(\delta_{1}+\gamma_{1}\right)+\alpha_{2}\left(\delta_{2}+\gamma_{2}\right) \leq 1, \\
& \alpha_{2} \gamma_{s} \leq 1, \\
& \alpha_{2} \gamma_{r} \leq 1,
\end{aligned}
$$

where $I_{1}^{\prime}$ and $I_{2}^{\prime}$ are the updated rate equations that can be obtained by setting $\mu_{1}=\mu_{2}=\mu_{s}=0$ in (19), (20) and (21) 
so that,

$$
\begin{aligned}
R_{p} & \leq \alpha_{1} \log _{2}\left(1+\frac{g_{p r} P_{p} \gamma_{1}}{N+g_{p r} P_{p} \delta_{1}}\right)+\alpha_{1} \log _{2}\left(1+\frac{g_{p d} P_{p} \delta_{1}}{N}\right) \\
& +\alpha_{2} \log _{2}\left(1+\frac{g_{p d} P_{p} \delta_{2}}{N}\right)=I_{1}^{\prime}, \\
R_{p} & \leq \alpha_{1} \log _{2}\left(1+\frac{g_{p d} P_{p}\left(\delta_{1}+\gamma_{1}\right)}{N}\right) \\
& +\alpha_{2} \log _{2}\left(1+\frac{g_{p d} P_{p}\left(\delta_{2}+\gamma_{2}\right)+g_{r d} P_{r} \gamma_{r}+g_{s d} P_{s} \gamma_{s}+\Gamma_{r p s}}{N}\right)=I_{2}^{\prime},
\end{aligned}
$$

where $\Gamma_{r p s}=2 \sqrt{g_{r d} g_{p d} \gamma_{r} \gamma_{2} P_{r} P_{p}}+2 \sqrt{g_{r d} g_{s d} \gamma_{r} \gamma_{s} P_{r} P_{s}}+$ $2 \sqrt{g_{s d} g_{p d} \gamma_{s} \gamma_{2} P_{s} P_{p}}$. By writing the Lagrangian, we get

$$
\mathcal{L}(\omega)=-\sum_{i=1}^{5} \lambda_{i} C_{i}(\omega)
$$

where $C_{1}(\omega)=I_{1}^{\prime}-R_{p}, C_{2}(\omega)=I_{2}^{\prime}-R_{p}, C_{3}(\omega)=1-$ $\alpha_{1}\left(\delta_{1}+\gamma_{1}\right)-\alpha_{2}\left(\delta_{2}+\gamma_{2}\right), C_{4}(\omega)=1-\alpha_{2} \gamma_{r}$ and $C_{5}(\omega)=$ $1-\alpha_{2} \gamma_{s}$. Differentiating the Lagrangian, we obtain:

$$
\begin{aligned}
\frac{\partial \mathcal{L}}{\partial \delta_{1}} & =\frac{\lambda_{1} g_{p r}^{2} P_{p} \gamma_{1}}{\left(N+g_{p r} P_{p}\left(\delta_{1}+\gamma_{1}\right)\right)\left(N+g_{p r} P_{p} \delta_{1}\right)} \\
& -\frac{\lambda_{1} g_{p d}}{N+g_{p d} P_{p} \delta_{1}}-\frac{\lambda_{2} g_{p d}}{N+g_{p d} P_{p}\left(\delta_{1}+\gamma_{1}\right)}+\lambda_{3} \frac{\ln 2}{P_{p}}=0 \\
\frac{\partial \mathcal{L}}{\partial \gamma_{1}} & =-\frac{\lambda_{1} g_{p r}}{N+g_{p r}\left(\delta_{1}+\gamma_{1}\right) P_{p}}-\frac{\lambda_{2} g_{p d}}{N+g_{p d}\left(\delta_{1}+\gamma_{1}\right) P_{p}}+\lambda_{3} \frac{\ln 2}{P_{p}}=0 \\
\frac{\partial \mathcal{L}}{\partial \delta_{2}} & =-\frac{\lambda_{1} g_{p d}}{N+g_{p d} P_{p} \delta_{2}}-\frac{\lambda_{2} g_{p d}}{N \omega_{r p s}^{\prime}}+\frac{\lambda_{3} \ln 2}{P_{p}}=0 \\
\frac{\partial \mathcal{L}}{\partial \gamma_{2}} & =-\frac{\lambda_{2}}{\omega_{r p s}^{\prime}}\left[\frac{g_{p d}}{N}+\sqrt{\frac{g_{r d} g_{p d} \gamma_{r} P_{r}^{p} P_{p}}{\gamma_{2} P_{p}}}+\sqrt{\frac{g_{s d} g_{p d} \gamma_{s} P_{s}^{p} P_{p}}{\gamma_{2} P_{p}}}\right] \\
& +\lambda_{3} \frac{\ln 2}{P_{p}}=0, \\
\frac{\partial \mathcal{L}}{\partial \gamma_{s}} & =-\frac{\lambda_{2}}{\omega_{r p s}^{\prime}}\left[\frac{g_{s d} P_{s}}{N}+\sqrt{\frac{g_{s d} g_{p d} \gamma_{2} P_{s}^{p} P_{p}}{\gamma_{s}}}+\sqrt{\frac{g_{s d} g_{r d} \gamma_{r} P_{s}^{p} P_{r}^{p}}{\gamma_{s}}}\right] \\
& +\lambda_{5} \ln 2=0,
\end{aligned}
$$

where $\omega_{r p s}^{\prime}=1+\frac{g_{p d} P_{p}\left(\delta_{2}+\gamma_{2}\right)+g_{r d} P_{r}^{p} \gamma_{r}+g_{s d} P_{s}^{p} \gamma_{s}+\Gamma_{r p s}}{N}$. We note that, similar to the previous problem, $\delta_{1}=0$ in order to avoid any inconsistency between equations (69) and (70). ${ }^{11}$

Also, $\lambda_{2}=0$ leads to a trivial problem, hence $\lambda_{2}>0$ which leads to $\lambda_{1}>0, \lambda_{3}>0$ and $\lambda_{5}>0$. Therefore, the first and second constraints are active and the primary and secondary power constraints are all active. From the first rate constraint, we have

$$
\gamma_{1}\left(\delta_{2}\right)=\frac{N}{g_{p r} P_{p}}\left[2^{\frac{R_{p}-\alpha_{2} \log _{2}\left(1+g_{p d} P_{p} \delta_{2} / N\right)}{\alpha_{1}}}-1\right],
$$

\footnotetext{
${ }^{11}$ We also know from the previous throughput problem that in order to maximize the secondary throughput, then $\gamma_{r}=1 / \alpha_{2}$, so we do not need to investigate the KKT conditions with respect to this variable.
}

while, from the primary power constraint, we get

$$
\gamma_{2}\left(\delta_{2}\right)=\frac{1-\alpha_{1} \gamma_{1}-\alpha_{2} \delta_{2}}{\alpha_{2}}
$$

(66) From the secondary source power constraint, we get,

$$
\gamma_{s}=1 / \alpha_{2}
$$

Now, using the values of (74), (75) and (76), we can find a solution to $\delta_{2}$ from the second rate equation such that

$$
\begin{aligned}
& g\left(\delta_{2}\right)=N+g_{p d} P_{p}\left(\delta_{2}+\gamma_{2}\left(\delta_{2}\right)\right)+g_{r d} P_{r}^{p} \gamma_{r}+g_{s d} P_{s}^{p} \gamma_{s}\left(\delta_{2}\right) \\
&+2 \sqrt{g_{r d} g_{s d} \gamma_{s}\left(\delta_{2}\right) \gamma_{r} P_{r}^{p} P_{s}^{p}}+2 \sqrt{g_{r d} g_{p d} \gamma_{2}\left(\delta_{2}\right) \gamma_{r} P_{p} P_{r}^{p}} \\
&+2 \sqrt{g_{p d} g_{s d} \gamma_{2}\left(\delta_{2}\right) \gamma_{s}\left(\delta_{2}\right) P_{p} P_{s}^{p}}-N \tau=0, \\
& \text { where } \tau=2^{\frac{R_{p}-\alpha_{1} \log _{2}\left(1+\frac{g_{p d} P_{p} \gamma_{1}\left(\delta_{2}\right)}{N}\right)}{\alpha_{2}}} \text {. We then calculate the }
\end{aligned}
$$
attainable throughput from each of the lowerbound problems and take their maximum as our lowerbound metric.

Next, we consider the case $\left|h_{p s}\right|>\left|h_{p d}\right|>\left|h_{p r}\right|$. In this case, relying on the secondary receiver is not beneficial for the primary. Hence, the communication scheme reduces to only using the secondary source as a relay. In the two cases of $\left|h_{p d}\right|>\left|h_{p s}\right|>\left|h_{p r}\right|$ or $\left|h_{p d}\right|>\left|h_{p r}\right|>\left|h_{p s}\right|$, direct transmission is the better option for the primary.

\section{APPENDIX C \\ Throughput Maximization With Total Power BUDGET}

A. The Case of $\left|h_{p s}\right|>\left|h_{p r}\right|>\left|h_{p d}\right|$

Herein, we also lowerbound the solution by transmitting only messages $m_{d}$ and $m_{r}$, i.e. $\mu_{1}=\mu_{2}=\mu_{s}=0$ as was previously done in Appendix B. The Lagrangian now can be written as,

$$
\mathcal{L}(\omega)=\left(1-\alpha_{1}-\alpha_{2}\right) \log _{2}\left(1+\frac{g_{s} \delta_{s} P_{s}}{N}\right)+\sum_{i=1}^{5} \lambda_{i} C_{i}(\omega),
$$

The analysis proceeds exactly as done in (69)-(72) with the only exception is that the differentiation of the Lagrangian with respect to $\gamma_{s}$ will be, ${ }^{12}$

$$
\begin{aligned}
\frac{\partial \mathcal{L}}{\partial \gamma_{s}} & =\frac{\lambda_{1}}{\omega_{r p s}^{\prime}}\left[\frac{g_{s d} P_{s}}{N}+\sqrt{\frac{g_{s d} g_{p d} \gamma_{2} P_{s} P_{p}}{\gamma_{s}}}+\sqrt{\frac{g_{s d} g_{r d} \gamma_{r} P_{s} P_{r}}{\gamma_{s}}}\right] \\
& -\frac{g_{s s} P_{s}}{\left(N+g_{s s} P_{s} \delta_{s}\right)\left(1-\alpha_{1}-\alpha_{2}\right)}-\lambda_{5} \ln 2=0,
\end{aligned}
$$

By solving the resulting equations together, we get the same values for $\gamma_{1}$ and $\gamma_{2}$ respectively as in (74) and (75). Using (72) in (70), we get

$$
\lambda_{2}=\lambda_{1} \frac{N+g_{p d} P_{p} \delta_{2}}{\omega_{r p s}^{\prime}}\left[\sqrt{\frac{g_{r d} P_{r} \gamma_{r}}{g_{p d} P_{p} \gamma_{2}}}+\sqrt{\frac{g_{s d} P_{s} \gamma_{s}}{g_{p d} P_{p} \gamma_{2}}}\right] .
$$

${ }^{12}$ Herein we will avoid introducing a new variable and substitute $\delta_{s}=$ $\frac{1-\alpha_{2} \gamma_{s}}{1-\alpha_{1}-\alpha_{2}}$. We also note that $C_{i}(\omega)$ will remain the same as the previous problem (65) 
Using (80) and (72) in (70), we get

$$
\begin{aligned}
& {\left[\sqrt{\frac{g_{r d} P_{r} \gamma_{r}}{g_{p d} P_{p} \gamma_{2}}}+\sqrt{\frac{g_{s d} P_{s} \gamma_{s}}{g_{p d} P_{p} \gamma_{2}}}\right]\left[\frac{g_{p d}}{\omega_{r p s}^{\prime}}-\frac{g_{p r}\left(N+g_{p d} P_{p} \delta_{2}\right)}{\omega_{r p s}^{\prime}\left(N+g_{p d} P_{p} \gamma_{1}\right)}\right]} \\
& +g_{p d}\left[\frac{1}{N+g_{p d} P_{p} \gamma_{1}}-\frac{1}{N \omega_{r p s}^{\prime}}\right]=0,
\end{aligned}
$$

so that $\gamma_{s}$ can be evaluated as

$$
\gamma_{s}\left(\delta_{2}\right)=\left(\frac{B-A \sqrt{\frac{g_{r d} P_{r} \gamma_{r}}{g_{p d} P_{p} \gamma_{2}}}}{A}\right)^{2} \frac{g_{p d} P_{p} \gamma_{2}}{g_{s d} P_{s}} .
$$

where $B=g_{p d}\left[\frac{1}{N+g_{p d} P_{p} \gamma_{1}}-\frac{1}{N \omega_{r p s}^{\prime}}\right]$ and $A=\frac{g_{p d}}{\omega_{r p s}^{\prime}}-$ $\frac{g_{p r}\left(N+g_{p d} P_{p} \delta_{2}\right)}{\omega_{r p s}^{\prime}\left(N+g_{p r} P_{p} \gamma_{1}\right)}$.

B. The Case of $\left|h_{p s}\right|>\left|h_{p d}\right|>\left|h_{p r}\right|$

Under these channel conditions, it is beneficial only to use node $\mathrm{S}$ for relaying the primary message. Hence, only message $m_{s}$ will be sent. Only two rate constraints are applicable

$$
\begin{aligned}
R_{p} & =\alpha_{1} \log _{2}\left(1+\frac{g_{p s} \mu_{1} P_{p}}{N+\delta_{1} g_{p s} P_{p}}\right)+\alpha_{1} \log _{2}\left(1+\frac{g_{p d} \delta_{1} P_{p}}{N}\right) \\
& +\alpha_{2} \log _{2}\left(1+\frac{g_{p d} \delta_{2} P_{p}}{N}\right)=I_{1}^{\prime \prime}, \\
R_{p} & =\alpha_{1} \log _{2}\left(1+\frac{g_{p d}\left(\delta_{1}+\mu_{1}\right) P_{p}}{N}\right) \\
& +\alpha_{2} \log _{2}\left(1+\frac{g_{p d}\left(\delta_{2}+\mu_{2}\right) P_{p}+g_{s d} \mu_{s} P_{s}+2 \sqrt{g_{p d} g_{s d} \mu_{2} \mu_{s} P_{p} P_{s}}}{N}\right)=I_{2}^{\prime \prime},
\end{aligned}
$$

where $I_{1}^{\prime \prime}$ and $I_{2}^{\prime \prime}$ can be recovered respectively from $I_{1}$ and $I_{2}$, by setting $\gamma_{1}=\gamma_{2}=\gamma_{s}=\gamma_{r}=0$. Then the throughput problem becomes

$$
\begin{aligned}
\max _{\omega} & \left(1-\alpha_{1}-\alpha_{2}\right) \log _{2}\left(1+\frac{g_{s} \delta_{s} P_{s}}{N}\right), \\
\text { s.t. } & I_{1}^{\prime \prime} \geq R_{p}, \quad I_{2}^{\prime \prime} \geq R_{p} \\
& R_{p} \geq \log _{2}\left(1+\frac{g_{p d} P_{p}}{N}\right) \\
& \alpha_{1}\left(\delta_{1}+\mu_{1}\right)+\alpha_{2}\left(\delta_{2}+\mu_{2}\right) \leq 1 \\
& \alpha_{2} \mu_{s} \leq 1
\end{aligned}
$$

where $\delta_{s}=\frac{1-\alpha_{2} \mu_{s}}{1-\alpha_{1}-\alpha_{2}}$. By writing the Lagrangian, we get

$$
\mathcal{L}(\omega)=\left(1-\alpha_{1}-\alpha_{2}\right) \log _{2}\left(1+\frac{g_{s} P_{s} \delta_{s}}{N}\right)+\sum_{i=1}^{4} \lambda_{i} C_{i}(\omega),
$$

where $C_{1}(\omega)=I_{1}^{\prime \prime}-R_{p}, C_{2}(\omega)=I_{2}^{\prime \prime}-R_{p}, C_{3}(\omega)=1-\alpha_{1}\left(\delta_{1}+\right.$ $\left.\mu_{1}\right)-\alpha_{2}\left(\delta_{2}+\mu_{2}\right)$ and $C_{4}(\omega)=1-\alpha_{2} \mu_{s}$. By differentiating the Lagrangian, it can be shown that the two rate constraints and the primary power constraint are active and we get an additional equation from the KKT conditions such that

$$
\mu_{1}\left(\delta_{2}\right)=\left(2^{\frac{R_{p}-\alpha_{2} \log _{2}\left(1+\frac{\delta_{2} g_{p d} P_{p}}{N}\right)}{\alpha_{1}}}-1\right) \frac{N}{g_{p s} P_{p}},
$$

$$
\begin{gathered}
\mu_{2}\left(\delta_{2}\right)=\frac{1-\alpha_{1} \mu_{1}-\alpha_{2} \delta_{2}}{\alpha_{2}}, \\
\mu_{s}\left(\delta_{2}\right)=\left(N \omega_{r p s}^{\prime \prime}[E+F]\right)^{2} \frac{\mu_{2} P_{p}}{g_{p d} g_{s d} P_{s}},
\end{gathered}
$$

where $E$ and $F$ are defined, respectively, as

$$
\begin{gathered}
E=\frac{g_{p s} g_{p d}}{N+\mu_{1} P_{p} g_{p s}}\left[\frac{\frac{1}{N \omega_{r p s}^{\prime \prime}}-\frac{1}{N+\mu_{1} P_{p} g_{p d}}}{\frac{g_{p s}}{N+\mu_{1} P_{p} g_{p s}}-\frac{g_{p d}}{N+\delta_{2} P_{p} g_{p d}}}\right], \\
F=\frac{g_{p d}}{N+\mu_{1} P_{p} g_{p d}}-\frac{g_{p d}}{N \omega_{r p s}^{\prime \prime}},
\end{gathered}
$$

where $\omega_{r p s}^{\prime \prime}=1+\frac{g_{p d}\left(\delta_{2}+\mu_{2}\right) P_{p}+g_{s d} \mu_{s} P_{s}+2 \sqrt{g_{p d} g_{s d} \mu_{2} \mu_{s} P_{p} P_{s}}}{N}$. We can then use these values in the following equation, which results from (84), to find the optimal value of $\delta_{2}$

$$
\begin{aligned}
f^{\prime \prime}\left(\delta_{2}\right)= & g_{p d} P_{p}\left(\delta_{2}+\mu_{2}\left(\delta_{2}\right)\right)+g_{s d} \mu_{s}\left(\delta_{2}\right) \\
& +2 \sqrt{g_{s d} g_{p d} \mu_{s}\left(\delta_{2}\right) \mu_{2}\left(\delta_{2}\right) P_{s} P_{p}}-\beta^{\prime \prime}=0, \\
\text { where } \beta^{\prime \prime}= & 2 \frac{R_{p-\alpha_{1} \log _{2}\left(1+\frac{g_{p d} P_{p} \mu_{1}\left(\delta_{2}\right)}{N}\right)}^{\alpha_{2}}}{\alpha_{2}}-1 .
\end{aligned}
$$

\section{The Case of $\left|h_{p r}\right|>\left|h_{p s}\right|>\left|h_{p d}\right|$}

In this case, node $\mathrm{R}$ sends $\left(m_{r}, m_{s}\right)$ while node $\mathrm{S}$ sends only $m_{s}$. The rate equations then become as shown in (93), (94), and (95). We will again lowerbound the problem by using,

$$
\begin{aligned}
R_{p} & \leq \alpha_{1} \log _{2}\left(1+\frac{g_{p s}\left(\gamma_{1}+\mu_{1}\right) P_{p}}{N+g_{p s} P_{p} \delta_{1}}\right)+\alpha_{1} \log _{2}\left(1+\frac{g_{p d} \delta_{1} P_{p}}{N}\right) \\
& +\alpha_{2} \log _{2}\left(1+\frac{g_{p d} \delta_{2} P_{p}}{N}\right)=\underline{I}_{1}^{\prime \prime \prime}
\end{aligned}
$$

We can show that $\delta_{1}=\mu_{2}=0$. Also, the first two rate constraints $\left(\underline{I}_{1}^{\prime \prime \prime}\right.$ and $\left.I_{2}^{\prime \prime \prime}\right)$ are active, along with the primary and node $\mathrm{R}$ power constraints, so that

$$
\begin{gathered}
\gamma_{1}+\mu_{1}=\frac{N}{g_{p r} P_{p}}\left[2^{\left.\frac{R_{p}-\alpha_{2} \log _{2}\left(1+g_{p d} P_{p} \delta_{2} / N\right)}{\alpha_{1}}-1\right]}\right. \\
\gamma_{2}=\frac{1-\alpha_{1}\left(\gamma_{1}+\mu_{1}\right)-\alpha_{2} \delta_{2}}{\alpha_{2}} .
\end{gathered}
$$

By differentiating the Lagrangian and writing the KKT conditions, we get

$$
\gamma_{r}=\frac{L^{2} \gamma_{2} P_{p}}{g_{r d} g_{p d} P_{r}}
$$

where

$$
L=N \omega_{r p s}^{\prime \prime \prime} \frac{\left[\frac{g_{p d}}{N \omega_{r p s}^{\prime \prime \prime}}-\frac{g_{p d}}{N+g_{p d}\left(\gamma_{1}+\mu_{1}\right) P_{p}}\right]}{\left[\frac{g_{p r}\left(N+g_{p d} P_{p} \delta_{2}\right)}{g_{p d}\left(N+g_{p r}\left(\gamma_{1}+\mu_{1}\right) P_{p}\right)}-1\right]} .
$$

with $\omega_{r p s}^{\prime \prime \prime}=1+\frac{g_{p d} P_{p}\left(\delta_{2}+\gamma_{2}+\mu_{2}\right)+g_{r d} P_{r}\left(\gamma_{r}+\mu_{r}\right)+g_{s d} P_{s} \mu_{s}+\zeta^{\prime \prime}}{N}$. 


$$
\begin{aligned}
R_{p} & \leq \alpha_{1} \log _{2}\left(1+\frac{g_{p s} \mu_{1} P_{p}}{N+g_{p s} P_{p}\left(\delta_{1}+\gamma_{1}\right)}\right)+\alpha_{1} \log _{2}\left(1+\frac{g_{p r} \gamma_{1} P_{p}}{N+g_{p r} P_{p} \delta_{1}}\right)+\alpha_{1} \log _{2}\left(1+\frac{g_{p d} \delta_{1} P_{p}}{N}\right)+\alpha_{2} \log _{2}\left(1+\frac{g_{p d} \delta_{2} P_{p}}{N}\right)=I_{1}^{\prime \prime \prime} \\
R_{p} & \leq \alpha_{1} \log _{2}\left(1+\frac{g_{p d} P_{p}\left(\delta_{1}+\gamma_{1}+\mu_{1}\right)}{N}\right) \\
& +\alpha_{2} \log _{2}\left(1+\frac{g_{p d} P_{p}\left(\delta_{2}+\gamma_{2}+\mu_{2}\right)+g_{r d} P_{r}\left(\gamma_{r}+\mu_{r}\right)+g_{s d} P_{s} \mu_{s}+\zeta^{\prime \prime}}{N}\right)=I_{2}^{\prime \prime \prime}, \\
R_{p} & \leq \alpha_{1} \log _{2}\left(1+\frac{g_{p s} P_{p} \mu_{1}}{N+g_{p s} P_{p}\left(\delta_{1}+\gamma_{1}\right)}\right)+\alpha_{1} \log _{2}\left(1+\frac{g_{p d} P_{p}\left(\delta_{1}+\gamma_{1}\right)}{N}\right) \\
& +\alpha_{2} \log _{2}\left(1+\frac{g_{p d} P_{p}\left(\delta_{2}+\gamma_{2}\right)+g_{r d} P_{r} \gamma_{r}+2 \sqrt{g_{p d} g_{r d} \gamma_{r} \gamma_{2} P_{p} P_{r}}}{N}\right)=I_{3}^{\prime \prime \prime},
\end{aligned}
$$

where $\zeta^{\prime \prime}=2 \sqrt{g_{r d} g_{p d} \gamma_{r} \gamma_{2} P_{r} P_{p}}+2 \sqrt{g_{r d} g_{s d} \mu_{r} \mu_{s} P_{r} P_{s}}+2 \sqrt{g_{s d} g_{p d} \mu_{s} \mu_{2} P_{s} P_{p}}+2 \sqrt{g_{r d} g_{p d} \mu_{r} \mu_{2} P_{r} P_{p}}$.

Furthermore, we have

$$
\mu_{s}=\frac{\mu_{r} g_{p d}^{2} g_{r d}}{g_{s d} P_{s} L^{2}}
$$

and

$$
\frac{g_{p d} \gamma_{2} P_{p}}{\gamma_{r}}=\frac{g_{s d} \mu_{s} P_{s}}{\mu_{r}} .
$$

Finally, using (97), (98), (99), (101) and (102), we can use the second rate equation to find a solution for $\delta_{2}$ such that

$$
\begin{aligned}
& \begin{array}{l}
k\left(\delta_{2}\right)= \\
\quad+2 \sqrt{g_{r d} g_{s d} \mu_{s}\left(\delta_{2}\right) \mu_{r}\left(\delta_{2}\right) P_{r} P_{s}}+2 \sqrt{g_{r d} g_{p d} \gamma_{2}\left(\delta_{2}\right) \gamma_{r}\left(\delta_{2}\right) P_{p} P_{r}} \\
\quad+g_{s d} P_{s} \gamma_{s}\left(\delta_{2}\right)-N \eta^{\prime \prime}=0,
\end{array} \\
& \text { where } \eta^{\prime \prime}=2 \frac{R_{p-\alpha_{1} \log _{2}\left(1+\frac{g_{p d} P_{p}\left(\gamma_{1}\left(\delta_{2}\right)+\mu_{1}\left(\delta_{2}\right)\right)}{N}\right)}^{\alpha_{2}}}{\alpha_{2}}
\end{aligned}
$$

\section{REFERENCES}

[1] Y.-C. Liang, K.-C. Chen, G. Y. Li, and P. Mähönen, "Cognitive radio networking and communications: An overview," IEEE Trans. Veh. Technol., vol. 60, no. 7, pp. 3386-3407, 2011.

[2] S. Srinivasa and S. A. Jafar, "The throughput potential of cognitive radio: A theoretical perspective," in Proc. Fortieth Asilomar Conf. Signals, Systems and Computers (ACSSC'06), Pacific Grove, CA, USA, Nov, 2006, pp. 221-225.

[3] Qing Zhao and B. Sadler, "A survey of dynamic spectrum access: Signal processing, networking, and regulatory policy," in IEEE Signal Processing Magazine, 2007, pp. 79-89.

[4] D. Hamza and S. Aissa, "Secondary access based on sensing and primary ARQ feedback in spectrum sharing systems," in IEEE Wireless Commun. Networking Conf. (WCNC'12), Paris, France, 2012, pp. 14841489.

[5] J. Gambini, O. Simeone, U. Spagnolini, and Y. Bar-Ness, "Cooperative cognitive radios with optimal primary detection and packet acceptance control," in Proc. IEEE Workshop Signal Process. Advances Wireless Commun. (SPAWC'07), Helsinki, Finland, June 2007, pp. 1-5.

[6] O. Simeone, J. Gambini, Y. Bar-Ness, and U. Spagnolini, "Cooperation and cognitive radio," in Proc. IEEE Int. Conf. on Commun. (ICC'07), Glasgow, Scotland, June 2007, pp. 6511-6515.

[7] S. H. Seyedmehdi and B. Liang, "Data rate and throughput analysis of cooperative cognitive radio under a collision model," in Proc. IEEE Conf. Comput. Commun. Workshops (INFOCOM WKSHPS), Shanghai, China, 2011, pp. 57-62.
[8] J. Zhang and Q. Zhang, "Stackelberg game for utility-based cooperative cognitiveradio networks," in Proc. Tenth ACM Int. Symp. Mobile and Ad Hoc Networking and computing (MobiHoc'09), New York, NY, USA, 2009 , pp. 23-32.

[9] T. M. Cover, "Comments on broadcast channels," IEEE Trans. Inform. Theory, vol. 44, no. 6, pp. 2524-2530, 1998.

[10] P. Popovski and E. de Carvalho, "Improving the rates in wireless relay systems through superposition coding," IEEE Trans. Wireless Commun., vol. 7, no. 12, pp. 4831-4836, 2008.

[11] A. K. Goparaju, S. Wei, and Y. Liu, "On superposition coding based cooperative diversity schemes," in Proc Thirty-Ninth Asilomar Conf. Signals, Systems and Computers, Pacific Grove, CA, USA, 2005, pp. 1046-1050.

[12] H. Kim, P. C. Cosman, and L. B. Milstein, , in Proc Fourty-Fourth Asilomar Conf. Signals, Systems and Computers,

[13] E.-H. Shin and D. Kim, "Time and power allocation for collaborative primary-secondary transmission using superposition coding," IEEE Commun. Lett., vol. 15, no. 2, pp. 196-198, 2011.

[14] J.-H. Wui and D. Kim, "Cognitive relaying systems based on network and superposition coding in multiple access primary channels," in IEEE Int. Conf. Signal Process., Commun. and Computing (ICSPCC'11), Xi' an, China, 2011, pp. 1-5.

[15] S. K. Jayaweera, M. Bkassiny, and K. A. Avery, "Asymmetric cooperative communications based spectrum leasing via auctions in cognitive radio networks," IEEE Trans. Wireless Commun., vol. 10, no. 8, pp. 2716-2724, Aug, 2011.

[16] D. Hamza and S. Aissa, "Enhanced primary and secondary performance through cognitive relaying and leveraging primary feedback," IEEE Trans. Veh. Technol., vol. PP, no. 99, pp. 1-1, Nov, 2013.

[17] W. Su, J. D. Matyjas, and S. Batalama, "Active cooperation between primary users and cognitive radio users in heterogeneous ad-hoc networks," IEEE Trans. Signal Process., vol. 60, no. 4, pp. 1796-1805, April 2012.

[18] D. Hamza, K.-H. Park, M.-S. Alouini, and S. Aissa, "Throughput maximization for cognitive radio networks using active cooperation and superposition coding," in Proc. IEEE Wireless Commun. Networking Conf. (WCNC'14), Istanbul, Turkey, 2014.

[19] Y. Chen, G. Yu, Z. Zhang, H.-H. Chen, and P. Qiu, "On cognitive radio networks with opportunistic power control strategies in fading channels," IEEE Trans. Wireless Commun., vol. 7, no. 7, pp. 27522761, July 2008.

[20] B. Wang, D. Zhao, and J. Cai, "Joint connection admission control and packet scheduling in a cognitive radio network with spectrum underlay," IEEE Trans. Wireless Commun., vol. 10, no. 11, pp. 3852 3863, November 2011.

[21] T. Cover and J. Thomas, Elements of Information Theory, John Wiley Inc., 1991.

[22] V. N. Q. Bao, T. Q. Duong, D. Benevides da Costa, G. C. Alexandropou- 
los, and A. Nallanathan, "Cognitive amplify-and-forward relaying with best relay selection in non-identical rayleigh fading," IEEE Commun. Lett., vol. 17, no. 3, pp. 475-478, 2013.

[23] Z. Beyaztas, A. Pandharipande, and D. Gesbert, "Optimum power allocation in a hierarchical spectrum sharing scheme," in Proc. IEEE Int. Conf. Commun. (ICC'08) Workshops, May 2008, pp. 97-101.

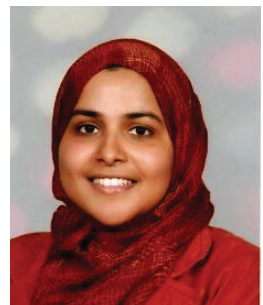

Doha Hamza (S'12,M'14) received the B.S. degree in electrical engineering from Alexandria University, Egypt, and the M.S. degree in wireless communications from Nile University, Egypt. She received her $\mathrm{PhD}$ in electrical engineering from King Abdullah University of Science and Technology (KAUST), Saudi Arabia in June 2014. Doha is currently a post doc with KAUST.

Her current research interests are dynamic spectrum access, cognitive radio networks, matching thetions, and cross-layer design. ory, game-theory application to wireless communica-

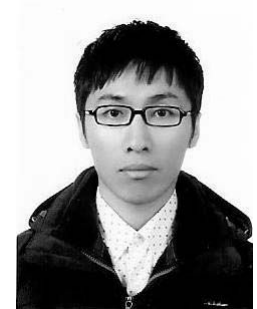

Ki-Hong Park (S'06, M'11) received his B.Sc. degree in Electrical, Electronic, and Radio Engineering from Korea University, Seoul, Korea, in 2005 and his M.S. and Ph.D. degrees in the School of Electrical Engineering from Korea University, Seoul, Korea, in 2011. Since April 2011, he has been a postdoctoral fellow of Electrical Engineering in the Division of Physical Science and Engineering at King Abdullah University of Science and Technology (KAUST), Thuwal, Saudi Arabia.

His research interests are broad in communication theory and its application to the design and performance evaluation of wireless communication systems and networks. On-going research includes the application to MIMO diversity/beamforming systems, cooperative relaying systems, physical layer secrecy, and optical wireless communications.

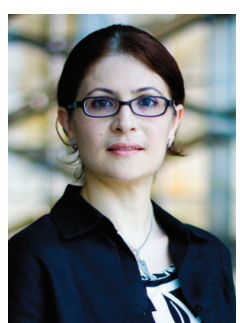

Sonia Aïssa (S'93-M'00-SM'03) received her Ph.D. degree in Electrical and Computer Engineering from McGill University, Montreal, QC, Canada, in 1998. Since then, she has been with the Institut National de la Recherche Scientifique-Energy, Materials and Telecommunications Center (INRS-EMT), University of Quebec, Montreal, QC, Canada, where she is a Full Professor

From 1996 to 1997, she was a Researcher with the Department of Electronics and Communications of Kyoto University, and with the Wireless Systems Laboratories of NTT, Japan. From 1998 to 2000, she was a Research Associate at INRS-EMT. In 2000-2002, while she was an Assistant Professor, she was a Principal Investigator in the major program of personal and mobile communications of the Canadian Institute for Telecommunications Research, leading research in radio resource management for wireless networks. From 2004 to 2007, she was an Adjunct Professor with Concordia University, Montreal. In 2006, she was Visiting Invited Professor with the Graduate School of Informatics, Kyoto University, Japan. Her research interests lie in the area of wireless and mobile communications, and include radio resource management, cross-layer design and optimization, design and analysis of multiple antenna (MIMO) systems, cognitive and cooperative transmission techniques, performance evaluation, and energy efficiency, with a focus on Cellular and Cognitive Radio networks.

Dr. Aïssa is the Founding Chair of the IEEE Women in Engineering Affinity Group in Montreal, 2004-2007; acted as TPC Leading Chair or Cochair of the Wireless Communications Symposium at IEEE ICC in 2006, 2009, 2011 and 2012; PHY/MAC Program Cochair of the 2007 IEEE WCNC; TPC Cochair of the 2013 IEEE VTC-spring; and TPC Symposia Cochair of the 2014 IEEE Globecom. Her main editorial activities include: Editor, IEEE Transactions on Wireless Communications, 2004-2012; Associate Editor, IEEE COMMUNICATIONS MAGAZINE, 2004-2009; Technical Editor, IEEE WIRELESS COMMUNICATIONS MAGAZINE, 2006-2010; and Associate Editor, Wiley Security and Communication Networks Journal, 2007-2012. She currently serves as Area Editor for the IEEE TRANSACTIONS ON WIRELESS COMMUNiCATIONS, and Technical Editor for the IEEE COMMUNICATIONS MAGAZINE. Awards to her credit include the NSERC University Faculty Award in 1999; the Quebec Government FQRNT Strategic Faculty Fellowship in 2001-2006; the INRS-EMT Performance Award multiple times since 2004, for outstanding achievements in research, teaching and service; and the Technical Community Service Award from the FQRNT Centre for Advanced Systems and Technologies in Communications in 2007. She is co-recipient of five IEEE Best Paper Awards and of the 2012 IEICE Best Paper Award; and recipient of NSERC Discovery Accelerator Supplement Award. She is a Distinguished Lecturer of the IEEE Communications Society (ComSoc) and

\section{an Elected Member of the ComSoc Board of Governors.}

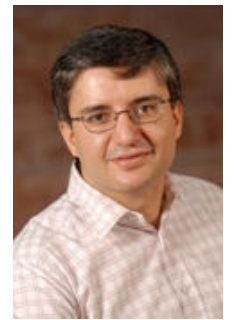

Mohamed-Slim Alouini (S'94, M'98, SM'03, F09) was born in Tunis, Tunisia. He received the Ph.D. degree in Electrical Engineering from the California Institute of Technology (Caltech), Pasadena, CA, USA, in 1998. He served as a faculty member in the University of Minnesota, Minneapolis, MN, USA, then in the Texas A\&M University at Qatar, Education City, Doha, Qatar before joining King Abdullah University of Science and Technology (KAUST), Thuwal, Makkah Province, Saudi Arabia as a Professor of Electrical Engineering in 2009. His current research interests include the modeling, design, and performance analysis of wireless communication systems. 